\title{
ALGEBRA EXTENSIONS AND NONSINGULARITY
}

\author{
JOACHIM CUNTZ AND DANIEL QUILLEN
}

This paper is concerned with a notion of nonsingularity for noncommutative algebras, which arises naturally in connection with cyclic homology.

Let us consider associative unital algebras over the complex numbers. We call an algebra $A$ quasi-free, when it behaves like a free algebra with respect to nilpotent extensions in the sense that any homomorphism $A \rightarrow R / I$, where $I$ is a nilpotent ideal in $R$, can be lifted to a homomorphism $A \rightarrow R$. If we restrict to the category of finitely generated commutative algebras, then this lifting property characterizes smooth algebras, the ones corresponding to nonsingular affine varieties. In this way quasi-free algebras appear as noncommutative analogues of smooth algebras. Stretching the analogy, we can even regard quasi-free algebras as analogues of manifolds.

One of the aims of this paper is to develop the analogy further by showing that quasi-free algebras provide a natural setting for noncommutative versions of certain aspects of manifolds. To give an example, let us consider the analogue of an embedding: an extension $A=R / I$, where $A$ and $R$ are quasi-free algebras playing the role of the submanifold and ambient manifold respectively. In the manifold situation, $I / I^{2}$ is the module of linear functions on the normal bundle, and the symmetric algebra $S_{A}\left(I / I^{2}\right)$ is the algebra of polynomial functions. Now in passing from commutative to noncommutative algebras, the symmetric algebra of a module is replaced by the tensor algebra of a bimodule. Thus the tensor algebra $T=T_{A}\left(I / I^{2}\right)$ is the noncommutative analogue of the normal bundle. In this situation we prove a formal tubular neighborhood theorem, which asserts that $R$ and $T$ become isomorphic after adically completing with respect to the kernels of the canonical homomorphisms to $A$.

Another aspect of manifolds that extends to quasi-free algebras is the concept of connection on the tangent bundle. Connes [Co] has defined connections on modules using noncommutative differential forms. We extend his idea to bimodules so that a connection on a bimodule $E$ over $A$ yields a way to extend derivations on $A$ to the tensor algebra $T_{A}(E)$. In the case of the bimodule $\Omega^{1} A$, a connection exists exactly when $A$ is quasi-free. For such a connection we construct (on a formal level) the geodesic flow and exponential map.

Quasi-free algebras can be characterized cohomologically as the algebras having cohomological dimension $\leq 1$ with respect to Hochschild cohomology. This shows that quasi-free algebras form a very restricted class. The tensor product of two quasi-free algebras is not quasi-free in general unless one of the algebras

Received by the editors March 2, 1993.

1991 Mathematics Subject Classification. Primary 14A22, 58B30.

(C)1995 American Mathematical Society 0894-0347/95 $\$ 1.00+\$ .25$ per page 
is separable. Smooth commutative algebras, and their deformations such as universal enveloping algebras, are not quasi-free when the dimension is $>1$.

We mention that Shelter [Sh] has discussed this extension of smooth algebras to the noncommutative context with emphasis on algebras satisfying a polynomial identity.

Besides investigating quasi-free algebras, another purpose of this paper is to develop tools which will be applied to cyclic homology in a succeeding paper. An important role is played by certain algebras generated from an algebra $A$ by means of universal mapping properties. The most basic of these is the DG algebra $\Omega A$ of noncommutative differential forms [Co], [Ka]. In addition we have the algebras $R A$ of [Cu2] and $Q A=A * A$ of [CC], [Cu1], which are useful in studying extensions of $A$, especially nilpotent extensions of higher order. The algebra $R A$ provides a standard way $A=R A / I A$ of writing $A$ as a quotient of a free algebra, and the resulting extension of $A$ is universal with respect to extensions $A=R / I$ equipped with a linear lifting $\rho: A \rightarrow R$ respecting identity elements. This universal extension captures the information of a functorial nature inherent in the choice of such a lifting $\rho$. It is a remarkable result that $R A$ and $Q A$ can be constructed in a simple fashion from $\Omega A$ using the Fedosov product:

$$
\omega \circ \xi=\omega \xi-(-1)^{|\omega|} d \omega d \xi
$$

introduced by Fedosov $[\mathrm{F}]$ in connection with the index theorem.

This paper is organized as follows. In $\S 1$ we construct $\Omega A$ and show that $R A$ and $Q A$ are isomorphic to the algebras of even forms and all forms respectively under Fedosov product. We also give a Fedosov type description of a free product of two algebras. In $\S 2$ we generalize $\Omega A$ to the algebra of relative differential forms $\Omega_{S} A$ in the case of a homomorphism $S \rightarrow A$. We establish a noncommutative cotangent exact sequence relating $\Omega^{1} S$ and $\Omega^{1} A$ to the relative differentials $\Omega_{S}^{1} A$.

The third section begins with the relation between differential forms and Hochschild cohomology. We show the bimodule $\Omega^{n} A$ is universal with respect to normalized $n$-cocycles with values in bimodule, where the universal $n$-cocycle is

$$
(d \cup \stackrel{n}{n} \cup d)\left(a_{1}, \ldots, a_{n}\right)=d a_{1} \cdots d a_{n} \in \Omega^{n} A .
$$

Separable and quasi-free algebras are then introduced using Hochschild cohomology. An algebra is quasi-free exactly when the universal 2-cocycle $d \cup d$ is a coboundary. We discuss 1-cochains $\phi$ satisfying $-\delta \phi=d \cup d$, because they can be used to describe connections on $\Omega^{1} A$.

Separable algebras are the noncommutative analogues of etale commutative algebras. An algebra is separable exactly when the universal derivation $d$ is inner, and the possible ways of writing it as an inner derivation are relevant to the study of connections. In $\S 4$ we show there is a canonical way of doing this related to the trace on the regular representation. At the same time we review various characterizations of separable algebras over the complex numbers.

The fifth section is devoted to properties and examples of quasi-free algebras. In $\S 6$ we begin with the lifting properties of separable and quasi-free algebras 
with respect to nilpotent extensions, and more generally extensions obtained by adic completion. We then prove the tubular neighborhood theorem. We also describe finite-dimensional quasi-free algebras in terms of path algebras associated to quivers.

In $\S 7$ we examine the universal extension $A=R A / I A$ when $A$ is quasifree. The tubular neighborhood theorem in this case asserts the existence of an isomorphism between $\widehat{R} A$ and $\widehat{\Omega}^{e v} A$ (i.e., between even forms with Fedosov and ordinary product). Following an idea from Yang-Mills theory, we explicitly construct an isomorphism starting from a cochain whose boundary is the universal 2-cocycle. By a related method we construct, in the case of a separable algebra, a conjugacy between the two embeddings of $A$ into $\widehat{Q} A$.

Finally, in the last section we study connections, first for right modules, next for bimodules, and then in the case of $\Omega^{1} A$, where we define torsion and formally construct the geodesic flow and exponential map.

\section{NONCOMMUTATIVE DIFFERENTIAL FORMS}

If $A$ is an algebra, then we construct the differential graded (DG) algebra $\Omega A$ of noncommutative differential forms on $A$ as follows. Let $\bar{A}$ denote the quotient vector space $A / \mathbb{C}$ of $A$ by the scalar multiples of the identity; in the case of the zero algebra we have $\bar{A}=0$. Let

$$
\Omega^{n} A=A \otimes \bar{A}^{\otimes n}
$$

for $n \geq 0$ and $\Omega^{n} A=0$ for $n<0$, and let the symbol $\left(a_{0}, \ldots, a_{n}\right)$ denote the image of $a_{0} \otimes \cdots \otimes a_{n}$ in $\Omega^{n} A$. We have $\Omega^{0} A=A$ and $(a)=a$. by

On $\Omega A=\bigoplus_{n \in \mathbb{Z}} \Omega^{n} A$ we define an operator $d$ of degree one and a product

$$
\begin{gathered}
d\left(a_{0}, \ldots, a_{n}\right)=\left(1, a_{0}, \ldots, a_{n}\right), \\
\left(a_{0}, \ldots, a_{n}\right)\left(a_{n+1}, \ldots, a_{k}\right)=\sum_{i=0}^{n}(-1)^{n-i}\left(a_{0}, \ldots, a_{i} a_{i+1}, \ldots, a_{k}\right) .
\end{gathered}
$$

Proposition 1.1. (1) These formulas determine a $D G$ algebra structure on $\Omega A$, which is the unique one satisfying

$$
a_{0} d a_{1} \cdots d a_{n}=\left(a_{0}, \ldots, a_{n}\right) .
$$

(2) Given a DG algebra $\Gamma=\bigoplus_{n} \Gamma^{n}$ and a homomorphism $u: A \rightarrow \Gamma^{0}$, there exists a unique homomorphism of $D G$ algebras $u_{*}: \Omega A \rightarrow \Gamma$ which extends $u$. Proof. We begin with the uniqueness part of (1). The following identities hold in any DG algebra containing $A$ as an even degree subalgebra:

$$
\begin{gathered}
d\left(a_{0} d a_{1} \cdots d a_{n}\right)=d a_{0} d a_{1} \cdots d a_{n} \\
\left(a_{0} d a_{1} \cdots d a_{n}\right)\left(a_{n+1} d a_{n+2} \cdots d a_{k}\right) \\
=(-1)^{n} a_{0} a_{1} d a_{2} \cdots d a_{k}+\sum_{i=1}^{n}(-1)^{n-i} a_{0} d a_{1} \cdots d\left(a_{i} a_{i+1}\right) \cdots d a_{k} .
\end{gathered}
$$


Applying these in the case of a DG algebra structure on $\Omega A$ satisfying (4) we see that the differential and the product must be given by (2) and (3), proving uniqueness.

To prove the existence we define $d$ on $\Omega A$ by (2). Then $d^{2}=0$, making $\Omega A$ a complex. Let $\mathscr{E}=\bigoplus \mathscr{E}^{n}$, where $\mathscr{E}^{n}$ is the space of linear operators of degree $n$ from $\Omega A$ to itself. Then $\mathscr{E}$ is a DG algebra with multiplication given by composition and with differential given by

$$
[d, w]=d \cdot w-(-1)^{|w|} \cdot d,
$$

where $|w|$ denotes the degree of $w$.

Let $l: A \rightarrow \mathscr{E}^{0}$ be the homomorphism which associates to $a \in A$ the left multiplication operator

$$
l a\left(a_{0}, \ldots, a_{n}\right)=\left(a a_{0}, \ldots, a_{n}\right) .
$$

We define a map $l_{*}: \Omega A \rightarrow \mathscr{E}$ by

$$
l_{*}\left(a_{0}, \ldots, a_{n}\right)=l a_{0}\left[d, l a_{1}\right] \cdots\left[d, l a_{n}\right] .
$$

Using the identities (5), (6) in the case of the subalgebra $l(A) \subset \mathscr{E}$, we see that the image $\operatorname{Im}\left(l_{*}\right)$ of $l_{*}$ is a DG subalgebra of $\mathscr{E}$; it is clearly the DG subalgebra generated by $l(A)$.

Consider next the map $\mathscr{E} \rightarrow \Omega A, w \mapsto w(1)$. Since

$$
\begin{aligned}
{\left[d, l a_{i}\right]\left(1, a_{i+1}, \ldots, a_{n}\right) } & =d\left(a_{i}, a_{i+1}, \ldots, a_{n}\right)-l a_{i} d\left(1, a_{i+1}, \ldots, a_{n}\right) \\
& =\left(1, a_{i}, a_{i+1}, \ldots, a_{n}\right),
\end{aligned}
$$

we find

$$
l a_{0}\left[d, d a_{1}\right] \cdots\left[d, l a_{n}\right](1)=\left(a_{0}, \ldots, a_{n}\right) .
$$

This shows that the map $w \mapsto w(1)$ is a retraction (i.e., left-inverse) for $l_{*}$, hence $l_{*}$ is injective.

We now use the isomorphism $l_{*}: \Omega A \stackrel{\sim}{\rightarrow} \operatorname{Im}\left(l_{*}\right)$ to transport the DG algebra structure on the latter to $\Omega A$. Then (8) gives (4) finishing the proof of (1).

Given $\Gamma$ and $u: A \rightarrow \Gamma^{0}$ as in (2), we define $u_{*}: \Omega A \rightarrow \Gamma$ by

$$
u_{*}\left(a_{0} d a_{1} \cdots d a_{n}\right)=u a_{0} d\left(u a_{1}\right) \cdots d\left(u a_{n}\right) .
$$

The identities (5), (6) and their counterparts for the elements $u a_{i} \in \Gamma$ show that $u_{*}$ is a DG algebra homomorphism. It is clearly the unique DG algebra homomorphism extending $u$, proving (2).

The universal extension. If $\Omega$ is a DG algebra, then the Fedosov product [F] on $\Omega$ is defined by

$$
\omega \circ \xi=\omega \xi-(-1)^{|\omega|} d \omega d \xi .
$$

Here $|\omega|=n$ if $\omega$ is homogeneous of degree $n$, and the product in general is determined by linearity. The Fedosov product is associative, as one easily checks. It is compatible with the even-odd $\mathbb{Z} / 2$ grading on $\Omega$ and hence makes $\Omega$ into a superalgebra. Even when the DG algebra $\Omega$ is (super) commutative, 
the Fedosov product is usually not commutative, because $\omega$ and $d \omega$ have opposite parity.

We now apply this construction to the DG algebra $\Omega A$ of noncommutative differential forms on the algebra $A$. This gives a superalgebra which we are going to relate to certain universal algebras constructed from $A$.

By a based linear map $\rho: A \rightarrow R$, where $R$ is an algebra, we mean a linear map between the underlying vector spaces which carries the identity of $A$ to the identity of $R$. We define the curvature of $\rho$ to be the bilinear map

$$
\omega\left(a_{1}, a_{2}\right)=\rho\left(a_{1} a_{2}\right)-\rho\left(a_{1}\right) \rho\left(a_{2}\right) .
$$

It vanishes if either $a_{1}$ or $a_{2}$ is the identity, hence it can be viewed as a linear $\operatorname{map} \omega: \bar{A}^{\otimes 2} \rightarrow R$.

We now consider the universal algebra $R A$ generated by a based linear map from $A$. This algebra can be constructed as follows. Let $T(A)=\bigoplus_{n \geq 0} A^{\otimes n}$ be the tensor algebra of the underlying vector space of $A$, and define $R A$ to be the quotient algebra

$$
R A=T(A) / T(A)\left(1_{T}-1_{A}\right) T(A),
$$

where $1_{T}$ is the identity of $T(A)$ and $1_{A}$ is the identity of $A$ regarded as a tensor of degree one. Let $\hat{\rho}: A \rightarrow R A$ be the based linear map given by the inclusion of $A$ into $T(A)$ as tensors of degree one followed by the canonical surjection to $R A$. It is then clear that $R A$ has the following universal mapping property: Given a based linear map $\rho: A \rightarrow R$, there is a unique homomorphism $R A \rightarrow R$ sending $\hat{\rho}$ to $\rho$.

As an algebra $R A$ depends only on the underlying vector space of $A$ and the identity element. If we choose a basis for $A$ containing $1_{A}$, then $R A$ is the free algebra with the generators given by the basis elements different from the identity.

To obtain structure on $R A$ reflecting the product on $A$, we consider the canonical homomorphism $R A \rightarrow A$ sending $\hat{\rho}$ to the identity map of $A$, and we let $I A$ be its kernel. We then have an algebra extension $A=R A / I A$ for which $\hat{\rho}$ is a based linear lifting, i.e., a based linear map which is a section of the canonical surjection $R A \rightarrow A$. It is clear that this is the universal 'extension equipped with based linear lifting' of $A$; we will refer to $R A$ as simply the universal extension of $A$.

Let $\hat{\omega}$ be the curvature of $\hat{\rho}$.

Proposition 1.2. There is a canonical isomorphism given by

$$
\hat{\rho}\left(a_{0}\right) \hat{\omega}\left(a_{1}, a_{2}\right) \cdots \hat{\omega}\left(a_{2 n-1}, a_{2 n}\right) \leftrightarrow a_{0} d a_{1} \cdots d a_{2 n}
$$

between $R A$ and the algebra $\Omega^{e v} A$ of even forms equipped with the Fedosov product. Under this isomorphism the ideal $I A^{n}$ corresponds to $\bigoplus_{k \geq n} \Omega^{2 k} A$. The associated graded algebra $\operatorname{gr}_{I A} R A=\bigoplus I A^{n} / I A^{n+1}$ is isomorphic to the algebra $\Omega^{e v} A$ of even forms with the ordinary product.

Proof. We consider the based linear map $A \rightarrow \Omega^{e v} A$ given by the inclusion, and note that its curvature is $a_{1} a_{2}-a_{1} \circ a_{2}=d a_{1} d a_{2}$. Let $\Psi: R A \rightarrow \Omega^{e v} A$ be the corresponding homomorphism given by the universal property of $R A$. 
We have $\Psi \hat{\rho}(a)=a, \Psi \hat{\omega}\left(a_{1}, a_{2}\right)=d a_{1} d a_{2}$. Since Fedosov product coincides with ordinary product when one of the forms is closed, we have

$$
\Psi\left(\hat{\rho}\left(a_{0}\right) \hat{\omega}\left(a_{1}, a_{2}\right) \cdots \hat{\omega}\left(a_{2 n-1}, a_{2 n}\right)\right)=a_{0} d a_{1} \cdots d a_{2 n} .
$$

On the other hand, since $\Omega^{2 n} A=A \otimes \bar{A}^{\otimes 2 n}$ we have a well-defined map $\Phi$ : $\Omega^{e v} A \rightarrow R A$ given by

$$
\Phi\left(a_{0} d a_{1} \cdots d a_{2 n}\right)=\hat{\rho}\left(a_{0}\right) \hat{\omega}\left(a_{1}, a_{2}\right) \cdots \hat{\omega}\left(a_{2 n-1}, a_{2 n}\right) .
$$

Clearly $\Phi$ is a section of $\Psi$, so these maps will be inverse isomorphisms provided $\Phi$ is surjective. Since

$$
\begin{aligned}
& \hat{\rho}(a) \cdot \hat{\rho}\left(a_{0}\right) \hat{\omega}\left(a_{1}, a_{2}\right) \cdots \hat{\omega}\left(a_{2 n-1}, a_{2 n}\right) \\
& \quad=\hat{\rho}\left(a a_{0}\right) \hat{\omega}\left(a_{1}, a_{2}\right) \cdots \hat{\omega}\left(a_{2 n-1}, a_{2 n}\right)-\hat{\omega}\left(a, a_{0}\right) \hat{\omega}\left(a_{1}, a_{2}\right) \cdots \hat{\omega}\left(a_{2 n-1}, a_{2 n}\right),
\end{aligned}
$$

the image of $\Phi$ is closed under left multiplication by $\hat{\rho}(a)$ for all $a \in A$. Since these elements generate $R A$, the image of $\Phi$ is a left ideal containing 1 , so $\Phi$ is surjective and we obtain the desired isomorphism.

Let us now identify $R A$ and $\Omega^{e v} A$ by means of this isomorphism and compare $I A^{n}$ with $F^{n}=\bigoplus_{k \geq n} \Omega^{2 k} A$. Since $d a_{1} d a_{2} \in I A$, we have $\Omega^{2 k} A \subset I A^{n}$ for $k \geq n$, hence $F^{n} \subset I A^{n}$. Now $F^{p} F^{q} \subset F^{p+q}$ by the definition of Fedosov product, and we have $I A=F^{1}$, since the quotient of $R A$ by either ideal is $A$. Thus $I A^{n}=\left(F^{1}\right)^{n} \subset F^{n}$, which proves $I A^{n}=F^{n}$ as claimed. The assertion about the associated graded algebra is clear.

In the sequel it is convenient to identify $R A$ with the space $\Omega^{e v} A$ of even forms equipped with Fedosov product. The universal property then says that any based linear map $\rho: A \rightarrow R$ extends uniquely to a homomorphism $\rho_{*}$ : $R A \rightarrow R$. We have

$$
\rho_{*}\left(a_{0} d a_{1} \cdots d a_{2 n}\right)=\rho\left(a_{0}\right) \omega\left(a_{1}, a_{2}\right) \cdots \omega\left(a_{2 n-1}, a_{2 n}\right),
$$

where $\omega$ is the curvature of $\rho$.

The algebra $Q A$. We next consider the algebra $Q A$ (cf. [Cu1], [CC]), which is defined to be the free product $A * A$. There are thus two canonical homomorphisms $l, l^{\gamma}$ from $A$ to $Q A$ which are a universal pair of homomorphisms from $A$ to another algebra. The algebra $Q A$ has a canonical automorphism $x \mapsto x^{\gamma}$ of order two which interchanges $l$ and $l^{\gamma}$.

We recall that a superalgebra is an algebra $S$ equipped with a $\mathbb{Z} / 2$ grading $S=S^{+} \oplus S^{-}$compatible with multiplication, and that such a grading is equivalent to an automorphism $\gamma$ of order two, where $S^{ \pm}$are the \pm 1 eigenspaces of $\gamma$. Thus $Q A$ is naturally a superalgebra. In fact, $Q A$ is the superalgebra generated by the algebra $A$ in the sense that a homomorphism $u: A \rightarrow S$ from $A$ to the underlying algebra of a superalgebra $S$ induces a superalgebra homomorphism $Q A \rightarrow S$. In effect, the grading automorphism $\gamma$ of $S$ gives a second homomorphism $u^{\gamma}: A \rightarrow S$, and the pair $u, u^{\gamma}$ determines a homomorphism from the free product $Q A=A * A$ to $S$. 
For $a \in A$, let $p a, q a$ denote the even and odd components of $l a$ with respect to the $\mathbb{Z} / 2$ grading of $Q A$. We have

$$
\begin{aligned}
l a & =p a+q a, \\
l^{\gamma} a & =p a-q a, \\
p\left(a_{1} a_{2}\right) & =p a_{1} p a_{2}+q a_{1} q a_{2}, \\
q\left(a_{1} a_{2}\right) & =p a_{1} q a_{2}+q a_{1} p a_{2} .
\end{aligned}
$$

Let $\mathbf{q} A$ be the kernel of the 'folding' homomorphism $Q A \rightarrow A$ which sends $l a, l^{\gamma} a$ to $a$. It is the ideal in $Q A$ generated by the elements $q a$ for $a \in A$.

Proposition 1.3. There is a canonical superalgebra isomorphism given by

$$
p a_{0} q a_{1} \cdots q a_{n} \leftrightarrow a_{0} d a_{1} \cdots d a_{n}
$$

between $Q A$ and the superalgebra $\Omega A$ of differential forms under Fedosov product. Under this isomorphism $\mathbf{q} A^{n}$ corresponds to $\bigoplus_{k \geq n} \Omega^{k} A$, and the associated graded algebra is isomorphic to $\Omega A$ with the usual multiplication of forms.

Proof. Since

$$
\begin{aligned}
\left(a_{1}+d a_{1}\right) \circ\left(a_{2}+d a_{2}\right) & =a_{1} a_{2}-d a_{1} d a_{2}+a_{1} d a_{2}+d a_{1} a_{2}+d a_{1} d a_{2} \\
& =a_{1} a_{2}+d\left(a_{1} a_{2}\right),
\end{aligned}
$$

we have a homomorphism from $A$ to $\Omega A$ equipped with Fedosov product given by $a \mapsto a+d a$. By the universal property of $Q A$ this extends to a superalgebra homomorphism $\Psi: Q A \rightarrow \Omega A$ such that $\Psi(p a)=a, \Psi(q a)=d a$, hence

$$
\Psi\left(p a_{0} q a_{1} \cdots q a_{n}\right)=a_{0} d a_{1} \cdots d a_{n} .
$$

On the other hand we have a section $\Phi: \Omega A \rightarrow Q A$ of $\Psi$ given by

$$
\Phi\left(a_{0} d a_{1} \cdots d a_{n}\right)=p a_{0} q a_{1} \cdots q a_{n},
$$

so $\Phi$ and $\Psi$ will be inverse isomorphisms if we show $\Phi$ is surjective. As $p 1=1$ we see from

$$
\begin{aligned}
& p a \cdot p a_{0} q a_{1} \cdots q a_{n}=p\left(a a_{0}\right) q a_{1} \cdots q a_{n}-q a q a_{0} q a_{1} \cdots q a_{n}, \\
& q a \cdot p a_{0} q a_{1} \cdots q a_{n}=q\left(a a_{0}\right) q a_{1} \cdots q a_{n}-p a q a_{0} q a_{1} \cdots q a_{n}
\end{aligned}
$$

that the image of $\Phi$ is closed under left multiplication by $p a, q a$. Since the elements $p a, q a$ for $a \in A$ generate $Q A$, the image of $\Phi$ is a left ideal which contains 1 , so $\Phi$ is surjective, and we have the desired isomorphism.

The assertion about ideals and the associated graded algebra follows in the same way as in the case of $R A$.

The free product $S * T$. Let us consider two algebras $S, T$, and use $x$ 's to denote elements of $S$ and $y$ 's for elements of $T$. We form the free product algebra $S * T$, and let $J$ be the ideal generated by commutators $[x, y], x \in S$, $y \in T$. Then $(S * T) / J$ can be identified with the tensor product algebra $S \otimes T$ in such a way that $x \otimes 1$ and $1 \otimes y$ correspond respectively to the classes of $x$ and $y$ modulo $J$. 
We consider the graded algebra

$$
\Gamma=\bigoplus_{n \geq 0} \Omega^{n} S \otimes \Omega^{n} T
$$

with the Fedosov type product defined by

$$
\left(\xi_{0} \otimes \eta_{0}\right) \circ\left(\xi_{1} \otimes \eta_{1}\right)=\xi_{0} \xi_{1} \otimes \eta_{0} \eta_{1}-(-1)^{\left|\xi_{0}\right|} \xi_{0} d \xi_{1} \otimes d \eta_{0} \eta_{1} .
$$

It is straightforward to check the associativity of this product.

Proposition 1.4. One has an algebra isomorphism $S * T \simeq \Gamma$ given by

$$
x_{0} y_{0}\left[x_{1}, y_{1}\right] \cdots\left[x_{n}, y_{n}\right] \leftrightarrow\left(x_{0} d x_{1} \cdots d x_{n}\right) \otimes\left(y_{0} d y_{1} \cdots d y_{n}\right) .
$$

Under this isomorphism $J^{n}$ corresponds to $\bigoplus_{k \geq n} \Omega^{k} S \otimes \Omega^{k} T$.

Proof. We have homomorphisms $x \mapsto x \otimes 1, y \mapsto 1 \otimes y$ from $S, T$ to $\Gamma$. By the universal property of the free product, these extend to a homomorphism $\Psi: S * T \rightarrow \Gamma$. We have

$$
\begin{aligned}
\Psi([x, y]) & =(x \otimes 1) \circ(1 \otimes y)-(1 \otimes y) \circ(x \otimes 1) \\
& =x \otimes y-(x \otimes y-d x \otimes d y)=d x \otimes d y,
\end{aligned}
$$

hence

$$
\Psi\left(x_{0} y_{0}\left[x_{1}, y_{1}\right] \cdots\left[x_{n}, y_{n}\right]\right)=\left(x_{0} d x_{1} \cdots d x_{n}\right) \otimes\left(y_{0} d y_{1} \cdots d y_{n}\right) .
$$

We prove $\Psi$ is an isomorphism by showing that the obvious section of $\Psi$ :

$$
\left(x_{0} d x_{1} \cdots d x_{n}\right) \otimes\left(y_{0} d y_{1} \cdots d y_{n}\right) \mapsto x_{0} y_{0}\left[x_{1}, y_{1}\right] \cdots\left[x_{n}, y_{n}\right]
$$

is surjective. It suffices to show that the subspace of the free product spanned by the elements on the right is closed under left multiplication by any $x \in R$ and any $y \in S$. The former is clear, and the latter follows from the identity

$$
y \cdot x_{0} y_{0}=x_{0} y y_{0}-\left[x_{0}, y\right] y_{0}=x_{0}\left(y y_{0}\right)-\left[x_{0}, y y_{0}\right]+y\left[x_{0}, y_{0}\right] \text {. }
$$

The assertion about $J^{n}$ is proved by the same argument used in the case of $R A$.

\section{RELATIVE DIFFERENTIAL FORMS}

Let $S$ be an algebra. By an $S$-algebra we mean an algebra $A$ equipped with a homomorphism $S \rightarrow A$. Let $A / S$ denote the cokernel of this homomorphism as a map of bimodules over $S$. We define the space of relative differential forms of degree $n$ with respect to $S$ to be

$$
\Omega_{S}^{n} A=A\left[\otimes_{S}(A / S)\right]^{(n)}=A \otimes_{S}(A / S) \otimes_{S} \cdots \otimes_{S}(A / S),
$$

where the notation []$^{(n)}$ means the expression inside the brackets is repeated $n$ times. Clearly $\Omega_{S}^{n} A$ is the quotient space of $\Omega^{n} A$ defined by the relations

$$
\begin{aligned}
& \left(a_{0}, \ldots, a_{i-1} s, a_{i}, \ldots, a_{n}\right)=\left(a_{0}, \ldots, a_{i-1}, s a_{i}, \ldots, a_{n}\right), \\
& \left(a_{0}, \ldots, a_{i-1}, s, a_{i+1}, \ldots, a_{n}\right)=0
\end{aligned}
$$


for $S$ in the image of $S$ in $A$ and $1 \leq i \leq n$. The formulas (2), (3) for the differential and product in $\Omega A$ are easily seen to be compatible with these relations, so $\Omega_{S} A=\bigoplus_{n} \Omega_{S}^{n} A$ is a quotient DG algebra of $\Omega A$.

Clearly the DG algebra $\Omega_{S} A$ of relative forms depends only on the image of $S$ in $A$. In order to simplify the notation it is convenient to identify $S$ with this image, when this does not lead to confusion.

With this convention we have the following universal property for the DG algebra of relative forms.

Proposition 2.1. Given a $D G$ algebra $\Gamma=\bigoplus \Gamma^{n}$ and a homomorphism $u: A \rightarrow$ $\Gamma^{0}$ such that $d(u S)=0$, there exists a unique $D G$ algebra homomorphism $u_{*}: \Omega_{S} A \rightarrow \Gamma$ extending $u$.

Proof. From 1.1 we know there is a unique DG algebra homomorphism $u_{*}$ : $\Omega A \rightarrow \Gamma$ extending $u$ given by

$$
u_{*}\left(a_{0} d a_{1} \cdots d a_{n}\right)=u a_{0} d\left(u a_{1}\right) \cdots d\left(u a_{n}\right) .
$$

When $d(u S)=0$ it is easily seen that $u_{*}$ is compatible with the relations (17) defining the relative forms. Hence $u_{*}$ factors through the quotient algebra $\Omega_{S} A$, proving the desired universal property.

Let us call a DG algebra $\Gamma$ equipped with a homomorphism $S \rightarrow \Gamma^{0}$ whose image is killed by $d$ a $D G S$-algebra. Then the universal property says that $\Omega_{S} A$ is the universal $D G S$-algebra generated by the $S$-algebra $A$.

Corollary 2.2. The algebra of relative forms can be identified with the quotient of $\Omega A$ by the ideal generated by $d S$ :

$$
\Omega_{S} A=\Omega A / \Omega A d S \Omega A .
$$

This is clear since the ideal generated by $d S$ is closed under $d$, and hence the quotient of $\Omega A$ by this ideal is a DG $S$-algebra with the same universal property as $\Omega_{S} A$.

We next establish for relative differential forms some basic properties of differential forms.

We recall that if $M$ is an $A$-bimodule, then one can form its $n$-th tensor product

$$
T_{A}^{n}(M)=M\left[\otimes_{A} M\right]^{(n-1)}=M \otimes_{A} \stackrel{n}{\cdots} \otimes_{A} M
$$

and tensor algebra $T_{A}(M)=\bigoplus_{n \geq 0} T_{A}^{n}(M)$, where $T_{A}^{0}(M)=A$. The tensor algebra has the following universal property: Given an algebra $R$ and a pair of maps $u: A \rightarrow R, v: M \rightarrow R$ such that $u$ is an algebra homomorphism and $v$ is an $A$-bimodule map relative to $u$, then there exists a unique homomorphism $T_{A}(M) \rightarrow R$ restricting to $u$ on $A$ and $v$ on $M$.

Proposition 2.3. $\Omega_{S} A$ is canonically isomorphic to the tensor algebra of the bimodule $\Omega_{S}^{1} A$ over $A$.

Proof. The inclusion homomorphism $A \rightarrow \Omega_{S} A$ and $A$-bimodule map $\Omega_{S}^{1} A \rightarrow$ $\Omega_{S} A$ induce a homomorphism of graded algebras $w: T_{A}\left(\Omega_{S}^{1} A\right) \rightarrow \Omega_{S} A$. We 
have

$$
\Omega_{S}^{n-1} A \otimes_{A} \Omega_{S}^{1} A=\Omega_{S}^{n-1} A \otimes_{A}\left(A \otimes_{S} A / S\right)=\Omega_{S}^{n-1} A \otimes_{S}(A / S)=\Omega_{S}^{n} A
$$

which we can iterate to obtain an isomorphism of $\Omega_{S}^{n} A$ with the $n$-th tensor product of the bimodule $\Omega_{S}^{1} A$. It is straightforward to check that this isomorphism coincides with $w$ in degree $n$.

The next two results concern the $A$-bimodule $\Omega_{S}^{1} A$ of relative differentials. Let us call a derivation $D: A \rightarrow M$, where $M$ is an $A$-bimodule, an $S$ derivation when $D S=0$.

Proposition 2.4. $d: A \rightarrow \Omega_{S}^{1} A$ is a universal $S$-derivation.

Proof. We must show that, for any $A$-bimodule $M$ and $S$-derivation $D: A \rightarrow$ $M$ satisfying $D S=0$, there is a unique bimodule map $D_{*}: \Omega_{S}^{1} A \rightarrow N$ such that $D_{*} d=D$. Consider the DG algebra given by the semi-direct product algebra $A \oplus M$, graded with $A$ in degree zero and $M$ in degree one, and with differential given by $D$. It is a DG $S$-algebra, hence the universal property of $\Omega_{S} A$ gives a unique DG algebra homomorphism $\Omega_{S} A \rightarrow A \oplus N$ extending the identity on $A$. Such a DG algebra homomorphism is clearly equivalent to a bimodule map $D_{*}: \Omega_{S}^{1} A \rightarrow N$ such that $D_{*} d=D$, whence the result.

Proposition 2.5. One has an exact sequence of A-bimodules

$$
0 \rightarrow \Omega_{S}^{1} A \stackrel{j}{\rightarrow} A \otimes_{S} A \stackrel{m}{\rightarrow} A \rightarrow 0,
$$

where $j\left(a_{0} d a_{1}\right)=a_{0} a_{1} \otimes 1-a_{0} \otimes a_{1}$ and $m\left(a_{0} \otimes a_{1}\right)=a_{0} a_{1}$.

Proof. The multiplication map $m$ has the section $i(a)=a \otimes 1$. Hence the projection operator $1-i m$ on $A \otimes_{S} A$ identifies the cokernel of $i$ with the kernel of $m$. By right exactness of tensor product the cokernel of $i$ is $A \otimes_{S}(A / S)=$ $\Omega_{S}^{1} A$. Thus we have an isomorphism $\Omega_{S}^{1} \stackrel{\sim}{\rightarrow} \operatorname{Ker}(m)$ given by

$$
a_{0} d a_{1} \mapsto(1-i m)\left(a_{0} \otimes a_{1}\right)=a_{0} \otimes a_{1}-a_{0} a_{1} \otimes 1=-j\left(a_{0} d a_{1}\right)
$$

which yields the desired short exact sequence. Finally $j$ is an $A$-bimodule map because it is the bimodule map induced by the inner derivation $a \mapsto[a, 1 \otimes 1]=$ $a \otimes 1-1 \otimes a$.

We next discuss some examples of algebras of relative differential forms.

As a first example, we consider the tensor algebra $T=T_{S}(M)$ of the $S$ bimodule $M$.

Proposition 2.6. There is a canonical T-bimodule isomorphism $T \otimes_{S} M \otimes_{S} T \simeq$ $\Omega_{S}^{1} T$.

Proof. We use the universal property 2.4 of $\Omega_{S}^{1} T$ with respect to $S$-derivations. If $N$ is a $T$-bimodule, then an $S$-derivation $D: T \rightarrow N$ is equivalent to an $S$-algebra lifting $1+D: T \rightarrow T \oplus N$ into the semidirect product. We then have 
the following natural equivalences:

$$
\begin{aligned}
\left\{T \text {-bimodule maps } \Omega_{S}^{1} T \rightarrow N\right\} \\
\quad=\{S \text {-derivations } T \rightarrow N\} \\
\quad=\{S \text {-algebra liftings } T \rightarrow T \oplus N\} \\
\quad=\{S \text {-bimodule maps } M \rightarrow N\} \\
\quad=\left\{T \text {-bimodule maps } T \otimes_{S} M \otimes_{S} T \rightarrow N\right\},
\end{aligned}
$$

whence the result.

We can also determine $\Omega_{S} T$ by a similar method. Let $K(M)$ be the complex of $S$-bimodules with $M$ in degrees zero and one, and zero in other degrees, where the differential from degree zero to degree one is the identity map of $N$. The tensor powers $T_{S}^{n}(K(N))$ are naturally complexes of $S$-bimodules, and the tensor algebra $T_{S}(C(N))$ is naturally a DG $S$-algebra.

Proposition 2.7. The DG $S$-algebras $\Omega_{S} T$ and $T_{S}(K(M))$ are canonically isomorphic.

Proof. Let $\Gamma$ be a DG $S$-algebra. Using universal properties we have the equivalences

$$
\text { \{DG } \begin{aligned}
&\left.S \text {-algebra maps } T_{S}(K(M)) \rightarrow \Gamma\right\} \\
&=\{\text { DG } S \text {-bimodule maps } K(M) \rightarrow \Gamma\} \\
&=\left\{S \text {-bimodule maps } M \rightarrow \Gamma^{0}\right\} \\
&=\left\{S \text {-algebra maps } T_{S}(M) \rightarrow \Gamma^{0}\right\} \\
&=\left\{\text { DG } S \text {-algebra maps } \Omega_{S} T \rightarrow \Gamma\right\}
\end{aligned}
$$

which yield the result.

As a second example, we consider the tensor product algebra $S \otimes A$ of two algebras $S$ and $A$. If we identify $S$ and $A$ with the subalgebras $S \otimes 1$ and $1 \otimes A$ of $S \otimes A$ respectively, then $S \otimes A$ can be regarded as the universal algebra generated by $S$ and $A$ such that $s a=a s$ for all $s \in S, a \in A$.

Proposition 2.8. There is a canonical isomorphism $\Omega_{S}(S \otimes A) \simeq S \otimes \Omega A$ of DG S-algebras.

Proof. We first note that $S \otimes \Omega A$ is a DG $S$-algebra with $d(s \omega)=s d \omega$ for $s \in S, \omega \in \Omega A$. By the universal property of relative forms there is a unique DG algebra map $\Omega_{S}(S \otimes A) \rightarrow S \otimes \Omega A$ extending the identity on $S \otimes A$. On the other hand, in $\Omega_{S}(S \otimes A)$ we have $s a=a s$ and $s d a=d(s a)=d(a s)=d a s$, hence $S$ commutes with the image of the composition $\Omega A \rightarrow \Omega(S \otimes A) \rightarrow$ $\Omega_{S}(S \otimes A)$. Thus we obtain a DG algebra map $S \otimes \Omega A \rightarrow \Omega_{S}(S \otimes A)$ which extends the identity on $S \otimes A$, and consequently is an inverse for the map we have in the opposite direction.

Taking $S$ to be the algebra of matrices $M_{n} \mathbb{C}$, we find

$$
\Omega_{M_{n} \mathrm{C}}\left(M_{n} A\right)=M_{n}(\Omega A) .
$$


In other words, the relative differential forms for matrices over $A$ with respect to scalar matrices are just the matrix differential forms over $A$.

Finally suppose $S$ is a commutative algebra, and let $A$ be a noncommutative algebra 'over' $S$ considered as ground ring in the sense of algebraic geometry. This means that one is given a homomorphism $S \rightarrow A$ whose image is contained in the center of $A$. In this case $s d a=d(s a)=d(a s)=d a s$ in $\Omega_{S} A$, hence $S$ maps to the center of $\Omega_{S} A$, so $\Omega_{S} A$ is a DG algebra over $S$. It is clearly the universal DG algebra over $S$ generated by $A$.

For instance, let us consider $S=\mathbb{C} \times \mathbb{C}$, and let $e, e^{\perp}$ be the idempotents $(1,0),(0,1)$. An algebra $A$ over $S$ is a direct product:

$$
A \stackrel{\sim}{\rightarrow} e A \times e^{\perp} A, \quad a \mapsto\left(e a, e^{\perp} a\right)
$$

and in this way the category of algebras over $S$ is equivalent to the category of pairs of algebras. A similar assertion holds for DG algebras, so it is clear that we have a canonical DG algebra isomorphism

$$
\Omega_{\mathbb{C} \times \mathbb{C}}\left(A_{1} \times A_{2}\right) \stackrel{\sim}{\rightarrow} \Omega A_{1} \times \Omega A_{2} .
$$

Cotangent sequence. Given a homomorphism $S \rightarrow A$, we have the following 'cotangent' exact sequence relating the relative and absolute differentials.

Proposition 2.9. One has a canonical exact sequence

$$
0 \rightarrow \operatorname{Tor}_{1}^{S}(A, A) \rightarrow A \otimes_{S} \Omega^{1} S \otimes_{S} A \rightarrow \Omega^{1} A \rightarrow \Omega_{S}^{1} A \rightarrow 0
$$

of bimodules over $A$.

Proof. The exact sequence of $S$-bimodules

$$
0 \rightarrow \Omega^{1} S \rightarrow S \otimes S \rightarrow S \rightarrow 0
$$

splits as a sequence of right modules, so tensoring over $S$ with $A$ on the right gives an exact sequence

$$
0 \rightarrow \Omega^{1} S \otimes_{S} A \rightarrow S \otimes A \rightarrow A \rightarrow 0 .
$$

We next tensor on the left with $A$. Since $S \otimes A$ is a free left $S$-module, the Tor long exact sequence yields an exact sequence

$$
0 \rightarrow \operatorname{Tor}_{1}^{S}(A, A) \rightarrow A \otimes_{S} \Omega^{1} S \otimes_{S} A \rightarrow A \otimes A \rightarrow A \otimes_{S} A \rightarrow 0 .
$$

From this we obtain the desired exact sequence, since $\Omega^{1} A, \Omega_{S}^{1} A$ are the kernels of the multiplication maps from $A \otimes A, A \otimes_{S} A$ to $A$ respectively.

Corollary 2.10. Let $T=T_{S}(M)$, where $M$ is an $S$-bimodule which is flat as either a left or right $S$-module. Then we have an exact sequence of $T$-bimodules

$$
0 \rightarrow T \otimes_{S} \Omega^{1} S \otimes_{S} T \rightarrow \Omega^{1} T \rightarrow T \otimes_{S} M \otimes_{S} T \rightarrow 0 .
$$

Proof. In view of the formula in Proposition 2.6 for $\Omega_{S}^{1} T$, it suffices to show that $\operatorname{Tor}_{1}^{S}(T, T)=0$. Assuming $M$ is right $S$-flat, the functor $X \mapsto M \otimes_{S} X$ on the category of $S$-modules is exact. Hence its $n$-th iterate

$$
\left[M \otimes_{S}\right]^{(n)} X=T_{S}^{n}(M) \otimes_{S} X
$$


is exact, which means that the $n$-th tensor power of the bimodule $M$ is right $S$-flat. Thus $T$ is right $S$-flat, and $\operatorname{Tor}_{1}^{S}(T, T)=0$. The case where $M$ is left flat is similar.

Corollary 2.11. Let $A=R / I$, where $I$ is an ideal in $R$. Then we have a short exact sequence of A-bimodules

$$
0 \rightarrow I / I^{2} \rightarrow A \otimes_{R} \Omega^{1} R \otimes_{R} A \rightarrow \Omega^{1} A \rightarrow 0,
$$

where the injection is induced by the restriction of the canonical derivation $d$ : $R \rightarrow \Omega^{1} R$ to $I$, and the surjection is induced by the canonical surjection $R \rightarrow A$. Proof. This results from cotangent sequence in the case of the surjection $R \rightarrow A$ and the canonical isomorphism $\operatorname{Tor}_{1}^{R}(A, A)=I / I^{2}$. To be more precise, let us consider the map of left $R$-module resolutions of $A$

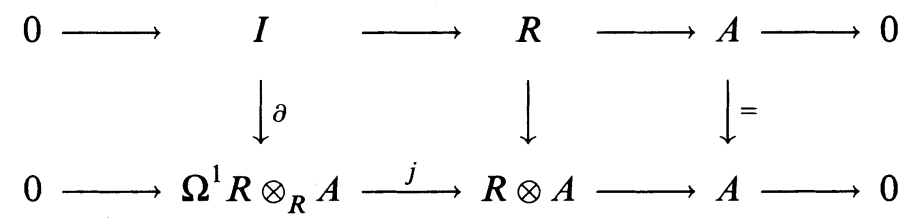

where the bottom row is $(20)$ and the vertical arrows are induced by the map $x \mapsto x \otimes 1$ from $R$ to $R \otimes A$. We have $j(x d y \otimes a)=x y \otimes a-x \otimes y a$ and $\partial z=d z \otimes 1$ for $z \in I$. Tensoring with $A$ on the left yields a map of exact sequences

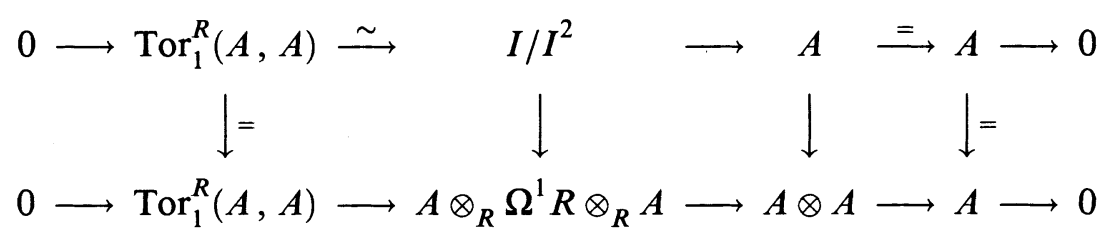

where the bottom row yields the cotangent exact sequence and the top row yields the canonical isomorphism just mentioned. The assertions about the maps in the exact sequence are now easily checked.

\section{HoCHSCHILD COHOMOLOGY}

In this section we discuss the relation between differential forms and Hochschild cohomology, and we define separable and quasi-free algebras.

The standard resolution. We consider the standard (normalized) resolution of $A$ by free bimodules

$$
\begin{gathered}
\stackrel{b^{\prime}}{\rightarrow} A \otimes \bar{A}^{\otimes 2} \otimes A \stackrel{b^{\prime}}{\rightarrow} A \otimes \bar{A} \otimes A \stackrel{b^{\prime}}{\rightarrow} A \otimes A \stackrel{b^{\prime}}{\rightarrow} A \rightarrow 0, \\
b^{\prime}\left(a_{0}, \ldots, a_{n+1}\right)=\sum_{i=0}^{n}(-1)^{i}\left(a_{0}, \ldots, a_{i} a_{i+1}, \ldots, a_{n+1}\right) .
\end{gathered}
$$

Let us make the identification

$$
\begin{aligned}
A \otimes \bar{A}^{\otimes n} \otimes A & =\Omega^{n} A \otimes A, \\
\left(a_{0}, \ldots, a_{n+1}\right) & \leftrightarrow a_{0} d a_{1} \cdots d a_{n} \otimes a_{n+1}
\end{aligned}
$$


and calculate the operator on $\Omega A \otimes A$ corresponding to $b^{\prime}$. Using the identity

$$
\begin{gathered}
a_{0} a_{1} d a_{2} \cdots d a_{n}+\sum_{i=1}^{n-1}(-1)^{i} a_{0} d a_{1} \cdots d\left(a_{i} a_{i+1}\right) \cdots d a_{n} \\
=(-1)^{n-1} a_{0} d a_{1} \cdots d a_{n-1} a_{n}
\end{gathered}
$$

when $n \geq 1$, the sum of the terms for $0 \leq i<n$ in (22) becomes

$$
(-1)^{n-1} a_{0} d a_{1} \cdots d a_{n-1} a_{n} \otimes a_{n+1}
$$

under our identification. Thus $b^{\prime}$ becomes

$$
\begin{aligned}
b^{\prime}\left(a_{0} d a_{1} \cdots d a_{n}\right)= & (-1)^{n-1} a_{0} d a_{1} \cdots d a_{n-1} a_{n} \otimes a_{n+1} \\
& +(-1)^{n} a_{0} d a_{1} \cdots d a_{n-1} \otimes a_{n} a_{n+1}
\end{aligned}
$$

which we can write simply

$$
b^{\prime}\left(\omega d a \otimes a^{\prime}\right)=(-1)^{|\omega|}\left(\omega a \otimes a^{\prime}-\omega \otimes a a^{\prime}\right) .
$$

We can therefore describe the standard resolution as the complex consisting of the free bimodules $\Omega^{n} A \otimes A$ for $n \geq 0$, and 0 for $n<0$, equipped with the differential $b^{\prime}$ given by (24). In addition there is the augmentation $m: A \otimes A \rightarrow A$ given by multiplication, which should be viewed as a map from this complex to $A$ viewed as a complex supported in degree zero. The augmentation is a quasi-isomorphism by the exactness of (21).

In fact the augmentation is a homotopy equivalence if either the left or right module structure is ignored. One proves this by means of two standard homotopies in which 1 is inserted on the left or on the right with the appropriate sign. In our differential form notation the homotopy equivalence compatible with right multiplication is given by the section $i(a)=1 \otimes a$ of $m$ together with the homotopy operator $d \otimes 1$ on $\Omega A \otimes A$. More precisely we have

$$
b^{\prime}(d \otimes 1)+(d \otimes 1) b^{\prime}=1
$$

in positive degrees and $b^{\prime}(d \otimes 1)+i m=1$ in degree zero. These formulas are easily verified using (24).

The homotopy equivalence compatible with left multiplication is given by the section $a \mapsto a \otimes 1$ of $m$ together with the homotopy operator on $\Omega A \otimes A$ given by

$$
\omega \otimes a \mapsto(-1)^{|\omega|+1} \omega d a \otimes 1 .
$$

A useful alternative method for showing that the augmentation is a quasiisomorphism is the following. The basic bimodule exact sequence 2.5

$$
0 \rightarrow \Omega^{1} A \stackrel{j}{\rightarrow} A \otimes A \stackrel{m}{\rightarrow} A \rightarrow 0
$$

splits as a sequence of left or right $A$-modules, so tensoring on the left with $\Omega^{n} A$ yields the bimodule exact sequence

$$
0 \rightarrow \Omega^{n+1} A \stackrel{j}{\rightarrow} \Omega^{n} A \otimes A \stackrel{m}{\rightarrow} \Omega^{n} A \rightarrow 0,
$$

where $j(\omega d a)=\omega a \otimes 1-\omega \otimes a, m(\omega \otimes a)=\omega a$. Comparing with (24) we 
have

$$
b^{\prime}=(-1)^{n} j m: \Omega^{n+1} A \otimes A \rightarrow \Omega^{n} A \rightarrow \Omega^{n} A \otimes A .
$$

We see therefore that, except for the sign of the differential, the standard resolution is obtained by splicing the short exact sequences (27) together.

Cochains. Recall that $A$-bimodules are the same as modules over the enveloping algebra $A^{e}=A \otimes A^{o p}$, and that the Hochschild cohomology groups of $A$ with coefficients the bimodule $M$ may be defined as

$$
H^{i}(A, M)=\operatorname{Ext}_{A^{e}}^{i}(A, M) .
$$

These Ext groups can be computed using the standard resolution of $A$, hence this cohomology is computed by the complex of (normalized) Hochschild cochains

with differential

$$
C^{n}(A, M)=\operatorname{Hom}_{A^{e}}\left(A \otimes \bar{A}^{\otimes n} \otimes A, M\right)=\operatorname{Hom}\left(\bar{A}^{\otimes n}, M\right)
$$

$$
\begin{aligned}
(\delta f)\left(a_{1}, \ldots, a_{n+1}\right)= & a_{1} f\left(a_{2}, \ldots, a_{n+1}\right) \\
& +\sum_{i=1}^{n}(-1)^{i} f\left(a_{1}, \ldots, a_{i} a_{i+1}, \ldots, a_{n+1}\right) \\
& +(-1)^{n+1} f\left(a_{1}, \ldots, a_{n}\right) a_{n+1} .
\end{aligned}
$$

From now on all cochains with values in a bimodule will be assumed normalized unless stated otherwise.

There is the following cup product operation on cochains. Given $f \in$ $C^{p}(A, M)$ and $g \in C^{q}(A, N)$ and a bimodule map $M \otimes_{A} N \rightarrow L, m \otimes n \mapsto$ $m \cdot n$, the cup product $f \cup g \in C^{p+q}(A, L)$ is defined by

$$
(f \cup g)\left(a_{1}, \ldots, a_{p+q}\right)=f\left(a_{1}, \ldots, a_{p}\right) \cdot g\left(a_{p+1}, \ldots, a_{p+q}\right) .
$$

We have

$$
\delta(f \cup g)=\delta f \cup g+(-1)^{|f|} f \cup \delta g,
$$

so the cup product of cocycles is a cocycle.

For example, the cochain

$$
\left(a_{1}, \ldots, a_{n}\right) \mapsto d a_{1} \cdots d a_{n} \in \Omega^{n} A
$$

is the $n$-fold cup product $d^{\cup n}$ of $d$. Since $d$ is a 1-cocycle (= derivation), it follows that $d^{\cup n}$ is an $n$-cocycle. In fact, $d^{\cup n}$ is the universal $n$-cocycle with values in a bimodule in the same way that $d$ is the universal 1-cocycle, as we now show.

Proposition 3.1. If $f$ is an $n$-cocycle with values in $M$, then there is a unique bimodule map $f_{*}: \Omega^{n} A \rightarrow M$ such that $f_{*}\left(d a_{1} \cdots d a_{n}\right)=f\left(a_{1}, \ldots, a_{n}\right)$.

Proof. There is a unique left $A$-module map $f_{*}$ with this property, so it suffices to show that $f_{*}$ is compatible with right multiplication if $f$ is a cocycle. Using the identity (23) with $n$ replaced by $n+1$ we have

$$
f_{*}\left(a_{0} d a_{1} \cdots d a_{n-1} a_{n}\right)-f_{*}\left(a_{0} d a_{1} \cdots d a_{n-1}\right) a_{n}=(-1)^{n} a_{0}(\delta f)\left(a_{1}, \ldots, a_{n+1}\right),
$$

so this is clear. 
We next introduce separable and quasi-free algebras using the properties of Hochschild cohomology in degrees $\leq 2$.

Given a bimodule $M$ over $A$, let

$$
M^{\natural}=\{x \in M \mid a x=x a, \forall a \in A\}
$$

denote its center. We have

$$
M^{\natural}=H^{0}(A, M)=\operatorname{Hom}_{A}^{e}(A, M) .
$$

We recall that $A$ is called separable when it has the equivalent properties listed in the following proposition.

Proposition 3.2. The following are equivalent:

(1) $A$ has cohomological dimension zero with respect to Hochschild cohomology.

(2) $A$ is a projective $A$-bimodule.

(3) Any derivation on $A$ with values in a bimodule is inner.

Proof. We recall that $H^{1}(A, M)$ can be identified with the space of derivations $D: A \rightarrow M$ modulo inner derivations. Thus (3) means that $\operatorname{Ext}_{A^{e}}{ }^{1}(A, M)=0$ for all $M$, which is equivalent to $A$ being a projective bimodule. In this case all the higher Ext groups vanish also, so the result is clear.

It will be useful when we study connections to understand the equivalence of (2) and (3) in concrete terms. It is clear by 2.4 that (3) is equivalent to the universal derivation $d: A \rightarrow \Omega^{1} A$ being inner, i.e., to the existence of an element $Y \in \Omega^{1} A$ such that

$$
d a=[a, Y], \quad a \in A,
$$

or equivalently $\delta Y=d$.

We consider the basic bimodule exact sequence

$$
0 \rightarrow \Omega^{1} A \stackrel{j}{\rightarrow} A \otimes A \stackrel{m}{\rightarrow} A \rightarrow 0,
$$

where $j\left(a_{0} d a_{1}\right)=a_{0}\left(a_{1} \otimes 1-1 \otimes a_{1}\right), m\left(a_{1} \otimes a_{2}\right)=a_{1} a_{2}$. Since $A \otimes A$ is the free bimodule with generator $1 \otimes 1$, it follows that $A$ is a projective bimodule iff this exact sequence splits.

A splitting is specified by a section $s$ of $m$ which is a bimodule map. By (34) $s$ is determined by $Z=s(1)$, where $Z$ can be any separability element, i.e., element $Z \in(A \otimes A)^{\natural}$ satisfying $m(Z)=1$. A splitting is also specified by a bimodule map $p$ which is a retraction (or left-inverse) for $j$. Now a bimodule map $p: A \otimes A \rightarrow \Omega^{1} A$ has the form

$$
p\left(a_{0} \otimes a_{1}\right)=a_{0} Y a_{1},
$$

where $Y=p(1 \otimes 1)$, and $p j=1$ means

$$
d a=p(a \otimes 1-1 \otimes a)=[a, Y]
$$

for all $a \in A$.

We thus see that the following data are equivalent:

(1) A bimodule splitting of the exact sequence (36). 
(2) A separability element.

(3) An element $Y \in \Omega^{1} A$ such that $\delta Y=d$.

In particular (2) and (3) in 3.2 are just different ways of saying that (36) splits.

We note that the section $s$ and retraction $p$ determine the same splitting when $j p+s m=1$. This means that the elements $Z, Y$ are related by

$$
1 \otimes 1=Z+j(Y) \text {. }
$$

If $Z=\sum x_{i} \otimes y_{i}$ where $z_{i}, y_{i} \in A$, then for $Z$ to be a separability element means

$$
\sum a x_{i} \otimes y_{i}=\sum x_{i} \otimes y_{i} a \quad \sum x_{i} y_{i}=1
$$

and we have $Y=\sum x_{i} d y_{i}$.

Square-zero extensions. By a square-zero extension of $A$ we mean an algebra extension $A=R / I$ such that $I^{2}=0$. In this situation $I$ is naturally a bimodule over $A$, and we can consider square-zero extensions of $A$ by a fixed bimodule $M$. A basic result about Hochschild cohomology identifies isomorphism classes of these extensions with elements of $H^{2}(A, M)$.

Proposition 3.3. The following conditions are equivalent:

(1) $A$ has cohomological dimension $\leq 1$ with respect to Hochschild cohomology.

(2) $\Omega^{1} A$ is a projective bimodule over $A$.

(3) For any square-zero extension $R$ of $A$ there is a lifting homomorphism $A \rightarrow R$.

$\operatorname{Proof}$ (see [Sh]). If $R$ is a square-zero extension of $A$ by $M$, then a lifting homomorphism $l: A \rightarrow R$ determines an isomorphism of $R$ with the semidirect product $A \oplus M$ such that, relative to this isomorphism, $l$ becomes the inclusion of $A$. Thus (3) means that every square-zero extension is a semidirect product, i.e., $H^{2}(A, M)=0$ for all bimodules $M$. From the basic exact sequence (26) and the long exact sequence of Ext groups we have

$$
H^{i+1}(A, M)=\operatorname{Ext}_{A^{e}}^{i+1}(A, M) \simeq \operatorname{Ext}_{A^{e}}^{i}\left(\Omega^{1} A, M\right)
$$

for $i>0$. Taking $i=1$, we see that $H^{2}(A, M)=0$ for all $M$ iff the bimodule $\Omega^{1} A$ is projective. The rest is clear.

Definition. We call $A$ quasi-free when it satisfies the conditions of the above proposition.

A free algebra is quasi-free, because for any extension of it we obtain a lifting homomorphism by lifting the generators and then extending this to a homomorphism. A separable algebra is quasi-free by the first conditions in 3.2 and 3.3.

For separable algebras one has a uniqueness result for the lifting homomorphism. Suppose given a square-zero extension $A=R / M$ and two lifting homomorphisms $l, l^{\prime}: A \rightarrow R$. Using the first we can identify $R$ with $A \oplus M$ so 
that $l a=a$. The second is then of the form $l^{\prime} a=a+D a$, where $D: A \rightarrow M$ is a derivation, which can be arbitrary. From the identity

$$
(1+m)^{-1} a(1+m)=a+[a, m]
$$

we see that $D$ is an inner derivation iff $l, l^{\prime}$ are conjugate by an element of $1+M$. Thus separable algebras are characterized by the property that any two lifting homomorphisms in a square-zero extension are conjugate by an element congruent to one modulo the ideal.

We next wish to understand the equivalence of (2) and (3) in 3.3 on a concrete level. We begin by relating (3) to the universal extension $R A$.

Let $A=R / M$ be a square-zero extension, and let $\rho: A \rightarrow R$ be a based linear lifting. By the universal property of $R A, \rho$ extends to a homomorphism $R A \rightarrow R$ of extensions of $A$. The kernel of this homomorphism contains $I A^{2}$, hence $\rho$ extends to a homomorphism $R A / I A^{2} \rightarrow R$ of square-zero extensions. In this way we can regard $R A / I A^{2}$ as the universal 'square-zero extension equipped with based linear lifting' of $A$. Clearly a lifting homomorphism for $R A / I A^{2}$ gives rise to one for $R$. Thus (3) is equivalent to the existence of a lifting homomorphism for $R A / I A^{2}$.

Now from 1.2 we have

$$
R A / I A^{2}=A \oplus \Omega^{2} A
$$

with multiplication given by Fedosov product modulo forms of degree $>2$. A based linear lifting $A \rightarrow R A / I A^{2}$ has the form $a \mapsto a-\phi a$ with $\phi: \bar{A} \rightarrow \Omega^{2} A$ linear. Since

$$
\left(a_{1}-\phi a_{1}\right) \circ\left(a_{2}-\phi a_{2}\right)=a_{1} a_{2}-d a_{1} d a_{2}-a_{1} \phi a_{2}-\phi a_{1} a_{2},
$$

this lifting is a homomorphism iff $\phi$ satisfies

$$
\phi\left(a_{1} a_{2}\right)=a_{1} \phi a_{2}+\phi a_{1} a_{2}+d a_{1} d a_{2}
$$

or equivalently $-\delta \phi=d \cup d$. Thus (3) is equivalent to the cocycle $d \cup d$ being a coboundary. This also can be seen from the fact that $d \cup d$ is the universal 2-cocycle. If it is a coboundary, then by functoriality every 2-cocycle is a coboundary, so $H^{2}(A, M)=0$ for all $M$.

Proposition 3.4. The following data are equivalent:

(1) A 1-cochain $\phi: \bar{A} \rightarrow \Omega^{2} A$ such that $-\delta \phi=d \cup d$.

(2) $A$ lifting homomorphism $A \rightarrow R A / I A^{2}$.

(3) An A-bimodule splitting of the exact sequence

$$
0 \rightarrow \Omega^{2} A \stackrel{j}{\rightarrow} \Omega^{1} A \otimes A \stackrel{m}{\rightarrow} \Omega^{1} A \rightarrow 0,
$$

where $j(\omega d a)=\omega a \otimes 1-\omega \otimes a, m(\omega \otimes a)=\omega a$.

(4) An operator $\nabla_{r}: \Omega^{1} A \rightarrow \Omega^{2} A$ satisfying

$$
\nabla_{r}(a \omega)=a \nabla_{r} \omega, \quad \nabla_{r}(\omega a)=\left(\nabla_{r} \omega\right) a+\omega d a .
$$

Proof. We have already proved the equivalence of (1) and (2).

A bimodule splitting of (40) is given by a retraction $p$ for $j$ which is a bimodule map. Since $\Omega^{1} A \otimes A=A \otimes \bar{A} \otimes A$, a bimodule map $p$ from $\Omega^{1} A \otimes A$ 
to $\Omega^{2} A$ is equivalent to a 1 -cochain $\phi: \bar{A} \rightarrow \Omega^{2} A$ via $p\left(a_{0} d a_{1} \otimes a_{2}\right)=a_{0} \phi a_{1} a_{2}$. We have

$$
\begin{aligned}
p j\left(d a_{1} d a_{2}\right) & =p\left(d a_{1} a_{2} \otimes 1-d a_{1} \otimes a_{2}\right) \\
& =p\left(d\left(a_{1} a_{2}\right) \otimes 1-a_{1} d a_{2} \otimes 1-d a_{1} \otimes a_{2}\right) \\
& =\phi\left(a_{1} a_{2}\right)-a_{1} \phi a_{2}-\phi a_{1} a_{2} .
\end{aligned}
$$

Thus $p j=1$ iff $\phi$ satisfies (39), proving the equivalence of (1) and (3).

A linear operator $\nabla_{r}: \Omega^{1} A \rightarrow \Omega^{2} A$ commuting with left multiplication is equivalent to a 1-cochain $\phi: \bar{A} \rightarrow \Omega^{2} A$ via $\nabla_{r}\left(a_{0} d a_{1}\right)=a_{0} \phi a_{1}$. We have

$$
\begin{gathered}
\nabla_{r}\left(a_{0} d a_{1} a\right)=\nabla_{r}\left(a_{0} d\left(a_{1} a\right)-a_{0} a_{1} d a\right)=a_{0} \phi\left(a_{1} a\right)-a_{0} a_{1} \phi a, \\
\nabla_{r}\left(a_{0} d a_{1}\right) a+a_{0} d a_{1} d a=a_{0} \phi a_{1} a+a_{0} d a_{1} d a,
\end{gathered}
$$

so $\nabla_{r}$ satisfies the Liebniz rule with respect to right multiplication iff $\phi$ satisfies (39). This proves the equivalence of (1) and (4).

Operators $\nabla_{r}$ as in (4) will be discussed below in the section on connections. Examples. 1. Consider a free algebra $A=T(V)$. In this case there is a canonical lifting homomorphism $A \rightarrow R A / I A^{2}$ extending the obvious lifting $v \mapsto v$ of the vector space of generators. The corresponding 1-cochain $\phi$ is determined by $\phi(v)=0$ for $v \in V$. We have

$$
\begin{aligned}
\phi\left(v_{1} \cdots v_{n}\right) & =\nabla_{r} d\left(v_{1} \cdots v_{n}\right) \\
& =\nabla_{r}\left(\sum_{i=1}^{n} v_{1} \cdots v_{i-1} d v_{i} v_{i+1} \cdots v_{n}\right) \\
& =\sum_{i=1}^{n-1} v_{1} \cdots v_{i-1} d v_{i} d\left(v_{i+1} \cdots v_{n}\right) .
\end{aligned}
$$

2. Suppose $A$ separable and let $Y$ be a 1-form such that $\delta Y=d$. The 1-cochains $(Y \cup d)(a)=Y d a$ and $(-Y \cup d)(a)=-Y d a$ both satisfy $-\delta \phi=$ $d \cup d$, as well as any affine linear combination.

\section{Separable algebras}

Our aim in this section is to show that a separable algebra $A$ has a canonical separability element, and hence a canonical 1-form $Y$ satisfying $d a=[a, Y]$. At the same time we review various more or less standard characterizations of separability. tion

Let $Z$ be an arbitrary separability element for $A$, and choose a representa-

$$
Z=\sum_{i=1}^{n} x_{i} \otimes y_{i}
$$

with $n$ least. Then $x_{1}, \ldots, x_{n}$ are linearly independent, and similarly for $y_{1}, \ldots, y_{n}$. From the first condition of (38) we have $\sum a x_{i} f\left(y_{i}\right)=\sum x_{i} f\left(y_{i} a\right)$ for any linear functional $f$ on $A$. Since the $y_{i}$ are linearly independent, the numbers $f\left(y_{i}\right)$ can be assigned arbitrarily, so the subspace $V$ spanned by the 
$x_{i}$ is a left ideal in $A$. The condition $\sum x_{i} y_{i}=1$ shows that if $a x_{i}=0$ for all $i$, then $a=0$. Thus $A$ is faithfully represented by left multiplication on $V$, and so we find that $A$ is finite dimensional as a vector space.

On the other hand, as a consequence of the fact that the bimodule $A$ is a direct summand of $A \otimes A$, we have for any (left) $A$-module $M$ that $M=$ $A \otimes_{A} M$ is a direct summand of the free $A$-module

$$
A \otimes M=(A \otimes A) \otimes_{A} M .
$$

Thus any $A$-module is projective, and consequently, any left ideal is generated by an idempotent. By Wedderburn theory an algebra with this property is a finite product of matrix algebras over skewfields. Since $A$ is finite dimensional and $\mathbb{C}$ is algebraically closed, these skewfields must be $\mathbb{C}$, and we see that $A$ is a finite product of matrix algebras over $\mathbb{C}$.

Let $\tau(a)$ be the trace of $a$ in the regular representation, i.e., the trace of left multiplication by $a$ on the vector space $A$. In the case of the matrix algebra $M_{n} \mathbb{C}$, one can compute that $\tau(a)=n \operatorname{tr}(a)$, where $\operatorname{tr}$ is the usual matrix trace, and that $\operatorname{tr}\left(a_{1} a_{2}\right)$ is a nondegenerate bilinear form. Thus $\tau\left(a_{1} a_{2}\right)$ is nondegenerate in this case (here we use in an essential way the fact that our groundfield has characteristic zero). Because $A$ is a product of matrix algebras, it follows that the bilinear form $\tau\left(a_{1} a_{2}\right)$ on $A$ is nondegenerate.

We next use the nondegeneracy of $\tau\left(a_{1} a_{2}\right)$ to construct a canonical separability element for $A$. Choose a basis $\left\{x_{i}\right\}$ of $A$, and let $\left\{y_{i}\right\}$ be the dual basis with respect to the bilinear form $\tau\left(a_{1} a_{2}\right)$, so that $\tau\left(y_{j} x_{i}\right)=\delta_{j i}$. The element $Z=\sum x_{i} \otimes y_{i}$ of $A \otimes A$ is independent of the choice of basis. Since the bilinear form is symmetric, we have $\sum x_{i} \otimes y_{i}=\sum y_{i} \otimes x_{i}$; in other words $Z$ is symmetric in the sense that

$$
\sigma(Z)=Z,
$$

where $\sigma$ is the flipping automorphism of $A \otimes A$.

Let $a_{j i}$ denote the matrix of left multiplication by $a$ with respect to the basis $x_{i}$. We then have

$$
a_{j i}=\tau\left(y_{i} a x_{j}\right), \quad a x_{i}=\sum_{j} a_{j i} x_{j}, \quad y_{j} a=\sum_{i} a_{j i} y_{i},
$$

hence

$$
a\left(\sum x_{i} \otimes y_{i}\right)=\sum a_{j i} x_{j} \otimes y_{i}=\sum x_{j} \otimes a_{j i} y_{i}=\left(\sum x_{j} \otimes y_{j}\right) a
$$

showing $Z \in(A \otimes A)^{\natural}$. Using the definition of $\tau$ and its trace property we have

$$
\tau(a)=\sum a_{i i}=\sum \tau\left(y_{i} a x_{i}\right)=\tau\left(a \sum x_{i} y_{i}\right)
$$

hence $\sum x_{i} y_{i}=1$ by nondegeneracy. This demonstrates the existence of a symmetric separability element for $A$.

We next want to prove the uniqueness, so we consider an arbitrary symmetric separability element $Z=\sum x_{i} \otimes y_{i}$. Then we have

$$
\sum x_{i} a \otimes y_{i}=\sum x_{i} \otimes a y_{i}, \quad \sum y_{i} x_{i}=1
$$

in addition to $(38)$. 
We now use $Z$ to prove for any bimodule $N$ over $A$ that we have an isomorphism

$$
N^{\natural} \stackrel{\sim}{\rightarrow} N_{\natural}
$$

given by the inclusion of the center followed by the canonical surjection of $N$ onto its commutator quotient space $N_{\natural}=N /[A, N]$. Let $T$ be the linear operator on $N$ given by $T(n)=\sum x_{i} n y_{i}$. The conditions (38) imply $T(n) \in N^{\natural}$ and $T(n)=n$ if $n \in N^{\natural}$. The conditions (42) imply $T$ kills $[A, N]$ and that $T$ is the identity modulo $[A, N]$, since $T(n)-n \sum y_{i} x_{i}=\sum\left[x_{i}, n y_{i}\right] \in[A, N]$. Thus the map $N_{\natural} \rightarrow N^{\natural}$ induced by $T$ is inverse to the canonical map the other way.

Next, we use (43) to show there is a unique symmetric separability element. We first note that $A \otimes A$ has, in addition to the outside $A$-bimodule structure considered up to now, the inside bimodule structure given by $a\left(a_{1} \otimes a_{2}\right)=$ $a_{1} \otimes a a_{2}$ and $\left(a_{1} \otimes a_{2}\right) a=a_{1} a \otimes a_{2}$. We apply (43) to $A \otimes A$ equipped with the inside bimodule structure and denote the center by $A \otimes^{\natural} A$; the commutator quotient space can be identified with $A$ via the multiplication $m: A \otimes A \rightarrow A$. Then by our assumption we have an isomorphism

$$
A \otimes^{\natural} A \stackrel{\sim}{\rightarrow} A
$$

induced by $m$, and this is a bimodule isomorphism with respect to the outside structure, since the inside and outside structures commute. Consequently, there is a unique $Z \in A \otimes^{\natural} A$ satisfying $m(Z)=1$, and $Z$ is also central for the outside structure, since $m(Z)=1$ is central in $A$. Thus $Z$ is a separability element, and it is the unique one central with respect to the inside structure. To show it is symmetric, we consider $\sigma(Z)$. This is central for both inside and outside structures, hence by the above isomorphism it is determined by $m \sigma(Z) \in A^{\natural}$. But $A^{\natural} \simeq A_{\natural}$ and $m \sigma(Z) \equiv m(Z) \bmod$ commutators, so $\sigma(Z)=$ $Z$ and $Z$ is symmetric.

We have therefore proved

Proposition 4.1. A separable algebra $A$ has a unique symmetric separability element $Z$. One has $Z=\sum x_{i} \otimes y_{i}$, where $\left\{x_{i}\right\}$ is any basis for $A$ and $\left\{y_{i}\right\}$ is the dual basis with respect to the bilinear form $\tau\left(a_{1} a_{2}\right)$, where $\tau$ is the trace associated to the regular representation.

Examples. 1. If $A=M_{n} \mathbb{C}$, then

$$
Z=\frac{1}{n} \sum_{i, j=1}^{n} e_{i j} \otimes e_{j i}
$$

where $e_{i j}$ denotes the matrix with 1 in the $(i, j)$-th position and zero elsewhere.

2. If $A$ is the group algebra $\mathbb{C}[G]$ of the finite group $G$, then

$$
Z=\frac{1}{|G|} \sum_{g \in G} g \otimes g^{-1}
$$


We now collect the various properties of separable algebras that we have discussed. Inspection of the arguments we have given shows that these properties are equivalent, so we obtain

Proposition 4.2. Separable algebras are characterized by any of the following properties:

(1) $A$ is projective as an A-bimodule.

(2) Any derivation on $A$ with values in an A-bimodule is inner.

(3) $A$ is a finite product of matrix algebras.

(4) $A$ is finite dimensional as a vector space, and the bilinear form $\tau\left(a_{1} a_{2}\right)$ is nondegenerate, where $\tau$ is the trace associated to the regular representation.

(5) One has $N^{\natural} \stackrel{\sim}{\rightarrow} N_{\natural}$ for all A-bimodules $N$.

\section{QUASI-FREE ALGEBRAS}

The purpose of this section is to give some idea of the possible types of quasifree algebras. We begin by showing that the class of quasi-free algebras is rather restricted.

We recall that $A$ is called (left) hereditary when any submodule of a projective (left) module over $A$ is projective. This is equivalent to any module having a projective resolution of length one.

Proposition 5.1. A quasi-free algebra is hereditary.

Proof. Let $M$ be an $A$-module, and consider the exact sequence

$$
0 \rightarrow \Omega^{1} A \otimes_{A} M \rightarrow A \otimes M \rightarrow M \rightarrow 0
$$

of $A$-modules obtained by tensoring the basic exact sequence (36) with $M$. If $A$ is quasi-free, then the bimodule $\Omega^{1} A$ is projective, i.e., it is a direct summand of the free bimodule $A \otimes V \otimes A$ for some vector space $V$. Hence $\Omega^{1} A \otimes_{A} M$ is a projective module, because it is a direct summand of the free module $A \otimes V \otimes M$. Thus this exact sequence is a projective resolution of $M$ of length one, so $A$ is hereditary.

An important property of hereditary algebras is the following.

Proposition 5.2. If $I$ is an ideal in a hereditary algebra $R$, then the associated graded algebra $\operatorname{gr}_{I} R=\bigoplus I^{n} / I^{n+1}$ is the tensor algebra on the bimodule $I / I^{2}$ over $R / I$.

Proof. In general for a flat module $M$ over $R$ we have isomorphisms $I \otimes_{R} M \stackrel{\sim}{\rightarrow}$ $I M$ and $\left(I / I^{2}\right) \otimes_{R} M \stackrel{\sim}{\rightarrow} I M / I^{2} M$ given by multiplication. Since

$$
\left(I / I^{2}\right) \otimes_{R} M=\left(I / I^{2}\right) \otimes_{R / I}(R / I) \otimes_{R} M=\left(I / I^{2}\right) \otimes_{R / I}(M / I M),
$$

we have

$$
\left(I / I^{2}\right) \otimes_{R / I}(M / I M) \stackrel{\sim}{\rightarrow} I M / I^{2} M .
$$

Now in a hereditary algebra any left ideal is a projective module, and hence flat. Hence we can apply this isomorphism to $I^{n-1}$ to obtain

$$
\left(I / I^{2}\right) \otimes_{R / I}\left(I^{n-1} / I^{n}\right) \stackrel{\sim}{\rightarrow} I^{n} / I^{n+1} .
$$


By induction this implies that $I^{n} / I^{n+1}$ is the $n$-th tensor product of the bimodule $I / I^{2}$ over $R / I$, which proves the assertion.

To illustrate these results, let us consider a finitely generated commutative algebra $A$ which is quasi-free. The first proposition says that $A$ is regular of dimension $\leq 1$, and the second implies that the graded algebra associated to any maximal ideal is a polynomial algebra of dimension $\leq 1$. Either of these properties means that $A$ is smooth of dimension $\leq 1$, i.e., it corresponds to a variety whose components are points and nonsingular affine curves.

In fact, one knows from Koszul complex calculations [HKR] that a smooth (commutative) algebra of dimension $n$ has Hochschild cohomological dimension $n$, hence it is quasi-free iff $n \leq 1$. Thus an algebra which is nonsingular in the commutative category becomes singular in the noncommutative category when the dimension is $>1$. This is closely related to the fact that, in contrast to the situation for smooth algebras, the tensor product of two quasi-free algebras need not be quasi-free. The Hochschild cohomological dimension of a tensor product is the sum of the dimensions of the factors in general, so the tensor product of two quasi-free algebras can have Hochschild cohomological dimension 2.

The following lists ways to produce quasi-free algebras.

Proposition 5.3. (1) The free product of any family of quasi-free algebras is quasifree.

(2) If $\Sigma$ is a subset of the quasi-free algebra $A$, then the algebra $A\left[\Sigma^{-1}\right]$ obtained by formally adjoining the inverses of elements of $\Sigma$ is quasi-free. In particular the group algebra of a free group is quasi-free.

(3) Let $A$ be quasi-free and let $N$ be a projective bimodule over $A$. Then the tensor algebra $T_{A}(N)$ is quasi-free.

(4) If $S$ is separable and $A$ is quasi-free, then $S \otimes A$ is quasi-free. In particular, $M_{n} A=M_{n} \mathbb{C} \otimes A$ is quasi-free.

(5) The product $A_{1} \times A_{2}$ of two quasi-free algebras is quasi-free.

Proof. All of these can be proved by showing that any square-zero extension has a lifting homomorphism. To prove (3), let $R$ be a square-zero extension of $T_{A}(N)$. As $A$ is quasi-free, there is a lifting homomorphism $A \rightarrow R$, and $R$ thereby becomes a bimodule over $A$. As $N$ is a projective bimodule, we can lift the inclusion $N \rightarrow T_{A}(N)$ to an $A$-bimodule map $N \rightarrow R$. Then these liftings of $A, N$ combined with the universal property of the tensor algebra induce the desired lifting homomorphism $T_{A}(N) \rightarrow R$. Similar arguments yield (1) and (2).

To prove (4), let $R$ be a square-zero extension of $S \otimes A$, and identify $S, A$ with the subalgebras $S \otimes 1,1 \otimes A$ of $A \otimes S$. As $S$ is separable, hence quasifree, there is a lifting homomorphism $S \rightarrow R$. Let us consider the centralizer $H^{0}(S, R)$ of $S$ in $R$. As the functor $H^{0}(S, ?)$ is exact for a separable algebra, it follows that $H^{0}(S, R)$ is a subalgebra of $R$ mapping surjectively onto $S \otimes A$. Since $A$ is quasi-free, there is a lifting homomorphism $A \rightarrow H^{0}(S, R)$. We then have homomorphisms from $S$ and $A$ to $R$ whose images commute, and these yield the desired lifting homomorphism $S \otimes A \rightarrow R$. 
A similar argument proves (5); one first lifts the separable subalgebra $\mathbb{C} \times \mathbb{C} \subset$ $A_{1} \times A_{2}$ and considers its centralizer.

Actually it is quicker to prove (4) and (5) by showing that the algebra considered as bimodule over itself has a projective resolution of length one. Thus for $S \otimes A$ we tensor the basic exact sequence (36) with $S$, and for $A_{1} \times A_{2}$ we use the direct sum of the sequences (36) for $A_{1}$ and $A_{2}$.

We next discuss a somewhat exotic type of quasi-free algebra.

Proposition 5.4. The inductive limit of a countable system $\cdots \rightarrow A_{n} \rightarrow A_{n+1} \rightarrow$ $\cdots$ of separable algebras is quasi-free.

An example of this situation is the unital algebra obtained by adjoining the identity matrix to the nonunital algebra $M_{\infty} \mathbb{C}$ consisting of infinite complex matrices $\left(x_{i j}\right), i, j \geq 1$, with finitely many nonzero entries. Here $A_{n}$ is the subalgebra $M_{n} \mathbb{C}$ of matrices with support in $1 \leq i, j \leq n$ with the infinite identity matrix adjoined. Since $M_{n} \mathbb{C}$ is unital, $A_{n}$ is isomorphic to $C \times M_{n} \mathbb{C}$, hence it is separable.

Proof. We first examine the cotangent sequence 2.9 in the case of a homomorphism $S \rightarrow A$, where $S$ is separable. Since modules over a separable algebra are projective, this sequence has the form

$$
0 \rightarrow A \otimes_{S} \Omega^{1} S \otimes_{S} A \rightarrow \Omega^{1} A \rightarrow \Omega_{S}^{1} A \rightarrow 0 .
$$

As $S$ is separable, we can choose $Y \in \Omega^{1} S$ satisfying $d s=[s, Y]$. Then $a \mapsto d a-[a, Y]$ is an $S$-derivation, which by 2.4 induces a bimodule lifting of $\Omega_{S}^{1} A$ into $\Omega^{1} A$. Thus the cotangent sequence splits yielding an $A$-bimodule isomorphism

$$
\Omega^{1} A \simeq A \otimes_{S} \Omega^{1} S \otimes_{S} A \oplus \Omega_{S}^{1} A .
$$

We apply this to the homomorphism $A_{n-1} \rightarrow A_{n}$ and extend from bimodules over $A_{n}$ to bimodules over the inductive limit $A_{\infty}$ to obtain

$$
\begin{aligned}
A_{\infty} \otimes_{A_{n}} \Omega^{1} A_{n} \otimes_{A_{n}} A_{\infty} & \simeq A_{\infty} \otimes_{A_{n-1}} \Omega^{1} A_{n-1} \otimes_{A_{n-1}} A_{\infty} \\
& \oplus A_{\infty} \otimes_{A_{n}} \Omega_{A_{n-1}}^{1} A_{n} \otimes_{A_{n}} A_{\infty},
\end{aligned}
$$

whence

$$
\Omega^{1} A_{\infty}=\underline{\lim } A_{\infty} \otimes_{A_{n}} \Omega^{1} A_{n} \otimes_{A_{n}} A_{\infty} \simeq \bigoplus_{n} A_{\infty} \otimes_{A_{n}} \Omega_{A_{n-1}}^{1} A_{n} \otimes_{A_{n}} A_{\infty} .
$$

Now bimodules over a separable algebra $S$, being modules over the separable algebra $S \otimes S^{o p}$, are automatically projective. Thus the bimodules over $A_{\infty}$ in the above direct sum are projective, and so is $\Omega^{1} A_{\infty}$.

\section{FORMAL TUBULAR NEIGHBORHOOD THEOREM}

Lifting properties. We have seen that quasi-free algebras have the property that any square-zero extension has a lifting homomorphism, and that separable algebras have the additional property that any two lifting homomorphisms in a 
square-zero extension are conjugate by an element lying over the identity of the algebra.

By virtue of the fact that any nilpotent extension is a composition of squarezero extensions, these properties extend to nilpotent extensions, and more generally, to extensions obtained by adic completion, as we now show.

Let $I$ be an ideal in the algebra $R$, and consider $R$ as a filtered algebra with the $I$-adic filtration. The completion

$$
\widehat{R}={\underset{n}{n}}_{\lim } R / I^{n+1}
$$

is naturally a filtered algebra with the completed $\widehat{I}$-adic filtration

$$
\left(I^{k}\right)^{-}=\underset{n}{\lim } I^{k} / I^{n+1} \text {. }
$$

We have $R / I^{n+1}=\widehat{R} /\left(I^{n+1}\right)^{-}$, and $R$ and $\widehat{R}$ have the same associated graded algebra.

Let $R_{n}=R / I^{n+1}$.

Proposition 6.1. (1) Assume $A$ quasi-free. Then any homomorphism $u_{0}: A \rightarrow$ $R_{0}$ lifts to a homomorphism $u: A \rightarrow \widehat{R}$.

(2) Assume $A$ separable. Suppose given homomorphisms $u, u^{\prime}: A \rightarrow \widehat{R}$ and an invertible element $g_{0} \in R_{0}$ such that $g_{0} u_{0} g_{0}^{-1}=u_{0}^{\prime}$, where $u_{0}, u_{0}^{\prime}: A \rightarrow R_{0}$ denote the reduction of $u, u^{\prime} \bmod \widehat{I}$. Then there exists a lifting of $g_{0}$ to an invertible element $g \in \widehat{R}$ such that $g u g^{-1}=u^{\prime}$.

Proof. (1) It suffices to construct inductively a sequence of homomorphisms $u_{n}: A \rightarrow R_{n}$ starting with $u_{0}$ such that $u_{n}$ lifts $u_{n-1}$. As $R_{n} \rightarrow R_{n-1}$ is a square-zero extension, we reduce to proving the assertion in the case $I^{2}=0$. We form the pull-back of $R$ by $u_{0}$

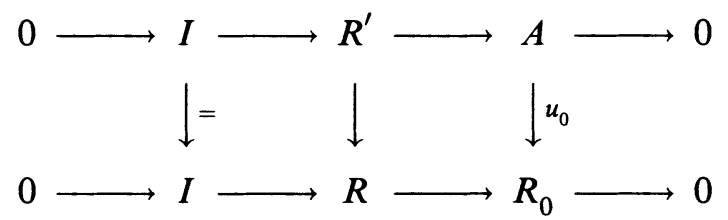

and note that a homomorphism $u$ lifting $u_{0}$ is equivalent to a lifting homomorphism $A \rightarrow R^{\prime}$. Since $R^{\prime}$ is a square-zero extension of $A$ and $A$ is quasi-free, this lifting homomorphism exists, yielding the desired homomorphism $u$.

(2) Let $u_{n}, u_{n}^{\prime}: A \rightarrow R_{n}$ be the reductions of $u, u^{\prime}$ modulo $\left(I^{n+1}\right)^{\wedge}$. It suffices to construct inductively a sequence of invertible elements $g_{n} \in R_{n}$ starting with $g_{0}$ such that $g_{n}$ lifts $g_{n-1}$ and $g_{n} u_{n} g_{n}^{-1}=u_{n}^{\prime}$. This reduces us to the case $I^{2}=0$. Let $h \in R$ be any lifting of $g_{0}$. Then $h$ is invertible, and after replacing $u$ by $h u h^{-1}$ we can suppose $u_{0}=u_{0}^{\prime}$ and $g_{0}=1$. Next we form the pull-back $R^{\prime}$ of $R$ by $u_{0}$ as above, and consider the two lifting homomorphisms $l, l^{\prime}: A \rightarrow R^{\prime}$ corresponding to $u, u^{\prime}$. As $A$ is separable, $l$ and $l^{\prime}$ are conjugate by an element of $1+I$, and hence so are $u$ and $u^{\prime}$. 
We now take up the tubular neighborhood theorem.

We consider an extension $A=R / I$, and let $N$ denote the $A$-bimodule $I / I^{2}$. Let $T=T_{A}(N)$ be the tensor algebra of $N$, let $J$ be the ideal in $T$ generated by $N$, so that $A=T / J$. We wish to compare the adic completions $\widehat{R}, \widehat{T}$ with respect to $I$ and $J$ respectively. These completions are filtered algebras with the completed adic filtrations. Since $T$ is graded, we have

$$
\widehat{T}=\prod_{n \geq 0} T_{A}^{n}(N), \quad \widehat{J}^{k}=\prod_{n \geq k} T_{A}^{n}(N)
$$

and the associated graded algebra of $\widehat{T}$ can be identified with $T$.

Theorem 1. Assume $A$ is quasi-free and $N$ is a projective A-bimodule. Then there is a surjective homomorphism $u: \widehat{T} \rightarrow \widehat{R}$ compatible with the completed adic filtrations, such that $\mathrm{gr}^{0} u, \mathrm{gr}^{1} u$ are the identity on $A, N$ respectively. Moreover, if $R$ is hereditary, then $u$ is an isomorphism.

Proof. As $A$ is quasi-free, there exists a lifting homomorphism $A \rightarrow \widehat{R}$ by 6.1. We can then view the canonical surjection $\widehat{I} \rightarrow N$ as a surjection of $A$ bimodules. As $N$ is projective, there exists an $A$-bimodule lifting $N \rightarrow \widehat{I}$. The pair of these liftings then induces a homomorphism $T \rightarrow \widehat{R}$, which carries the ideal $J$ into $\widehat{I}$, hence this homomorphism extends to the completion to give a homomorphism

$$
u: \widehat{T}=\prod_{n} T_{A}^{n}(N) \rightarrow \widehat{R}
$$

of filtered algebras. The map on associated graded algebras $\operatorname{gr}(u)$ can be identified with the homomorphism $T \rightarrow \operatorname{gr} R$ which is the identity in degrees 0,1 . Since $\operatorname{gr} R$ is generated by degrees 0,1 , we see that $\operatorname{gr}(u)$ is surjective, and a standard consequence of this and completeness is that $u$ is surjective. When $R$ is hereditary, we know that $\mathrm{gr} R$ is a tensor algebra by the lemma above, hence $\operatorname{gr}(u)$ and $u$ are isomorphisms.

The formal tubular neighborhood theorem is the following variant of this result.

Theorem 2. Assume $R$ and $A$ are quasi-free. Then there is an isomorphism of filtered algebras $u: \widehat{T} \rightarrow \widehat{R}$ such that $\mathrm{gr}^{0} u, \mathrm{gr}^{1} u$ are the identity on $A, N$ respectively.

Proof. Since $R$ is hereditary by 5.1 , this will follow from Theorem 1 once we show that $N$ is a projective bimodule over $A$. But this is a consequence of the cotangent exact sequence 2.11

$$
0 \rightarrow N \rightarrow A \otimes_{R} \Omega^{1} R \otimes_{R} A \rightarrow \Omega^{1} A \rightarrow 0
$$

since $\Omega^{1} R$ and $\Omega^{1} A$ are projective bimodules over $R$ and $A$ respectively.

We now give another proof using a construction which has a geometric interpretation. This construction will be applied later in the case of the universal extension. 
As $A$ is quasi-free, we know $R / I^{2}$ is isomorphic to the semi-direct product $A \oplus N$. We choose an isomorphism and consider the derivation on $R / I^{2}$ given by zero on $A$ and one on $I / I^{2}$. This gives a derivation $R \rightarrow N$ which can be lifted to a derivation $D: R \rightarrow I$, since $\Omega^{1} R$ is a projective $R$-bimodule. Then $D\left(I^{n}\right) \subset I^{n}$ for all $n$, hence $D$ induces a derivation on the associated graded algebra gr $R$. Since this derivation is zero on $A=R / I$ and one on $N=I / I^{2}$, it is $n$ on $I^{n} / I^{n+1}$. We then have

$$
(D-n) \cdots(D-1) D(R) \subset I^{n+1}
$$

by induction. It follows that $R / I^{n+1}$ decomposes into eigenspaces of $D$ corresponding to the eigenvalues $0,1, \ldots, n$, and, as $D$ is a derivation, this decomposition is a grading compatible with product. Thus we obtain an isomorphism of $R / I^{n+1}$ with its associated graded algebra by lifting $I^{k} / I^{k+1}$ to the eigenspace of $D$ on $I^{k} / I^{n+1}$ corresponding to the eigenvalue $k$. Taking the inverse limit as $n$ becomes infinite, we obtain an isomorphism of $\widehat{R}$ with the completion of $\operatorname{gr} R$. But $\operatorname{gr} R=T_{A}(N)$ by 5.1 and 5.2, which finishes the proof.

The geometric picture behind this result is the following. The extension $A=R / I$ with $R$ and $A$ quasi-free should be regarded as a noncommutative analogue of an embedding $Y \subset X$ of manifolds or nonsingular affine varieties. The tensor algebra $T=T_{A}(N)$ corresponds to the normal bundle $\mathscr{N}=\left(\left.T X\right|_{Y}\right) / T Y$ of the embedding, where $\left.T X\right|_{Y}$ denotes the restriction of the tangent bundle of $X$ to $Y$, and the isomorphism $\widehat{T} \simeq \widehat{R}$ can be interpreted as an isomorphism between the formal neighborhoods of $Y$ in $\mathscr{N}$ and $X$.

The construction in the second proof has the following geometric interpretation. The lifting homomorphism $A \rightarrow R / I^{2}$ corresponds to a lifting of $\mathscr{N}$ to a subbundle of $\left.T X\right|_{Y}$ complementary to $T Y$. The derivation $D$ corresponds to a vector field on $X$ vanishing on $Y$, whose 1 -jet along $Y$, viewed as an endomorphism of $\left.T X\right|_{Y}$, is the projection onto this complement. Such a vector field gives rise to a tubular neighborhood isomorphism in which the normal space at a point of $Y$ is the expanding submanifold for the vector field issuing from this point.

Finite dimensional quasi-free algebras. Let $S$ be a separable algebra, and let $N$ be an $S$-bimodule. Since $S$ is quasi-free and bimodules over a separable algebra are automatically projective, we know from 5.3(3) that the tensor algebra $T_{S}(N)$ is quasi-free.

Let us consider first the case where $S$ is commutative, that is, $S=\mathbb{C} e_{1} \oplus$ $\cdots \oplus \mathbb{C} e_{r}$, where the $e_{i}$ are orthogonal idempotents. We choose a basis $A_{i j}$ for $e_{i} N e_{j}$, and consider the quiver having the vertices $e_{i}$ and having $A_{i j}$ as the set of arrows from $e_{i}$ to $e_{j}$. Then $T_{S}(N)$ can be identified with the path algebra of this quiver (cf. [B], Chapter 4). The algebra $T_{S}(N)$ is finite dimensional (as a vector space) iff the quiver has finitely many edges and no oriented cycles.

In the general case, we can choose a commutative separable algebra $S^{\prime}$ which is Morita equivalent to $S$, and if $N^{\prime}$ is the $S^{\prime}$-bimodule corresponding to $N$, 
the algebras $T_{S}(N)$ and $T_{S^{\prime}}\left(N^{\prime}\right)$ are Morita equivalent. In this way algebras of the form $T_{S}(N)$ with $S$ separable are classified up to Morita equivalence by quivers (with finitely many vertices). The finite-dimensional algebras of this form correspond to quivers with finitely many vertices and arrows having no oriented cycles.

For instance, the algebra of triangular block matrices

$$
\left(\begin{array}{lll}
* & * & * \\
& * & * \\
& & *
\end{array}\right)
$$

is of the form $T_{S}(N)$, where $S$ is the (block) diagonal subalgebra, and $N$ consists of matrices supported in the diagonal just above the main diagonal. In this case the algebra $T_{S^{\prime}}\left(N^{\prime}\right)$ is the triangular matrix algebra of the same shape in which all the blocks are $1 \times 1$, and the quiver is:

Let $R$ be a finite dimensional algebra, let $I$ be the radical of $R$, and let $S=R / I$. One knows that $S$ is separable, and that $I$ is nilpotent. Applying Theorem 1, we obtain a surjective homomorphism $u: T_{S}(N) \rightarrow R$, where it is unnecessary to complete the tensor algebra here as $I$ is nilpotent. Furthermore, if $R$ is hereditary, then $u$ is an isomorphism, whence $R$ is quasi-free. As quasi-free algebras are hereditary, we conclude the following.

Proposition 6.2. A finite dimensional algebra is quasi-free iff it is hereditary. These algebras are the ones of the form $T_{S}(N)$ with $S$ separable such that the associated quiver has finitely many edges and no oriented cycles.

\section{UNIVERSAL LIFTINGS AND CONJUGACIES}

We recall our identification of $R A$ with the algebra of even differential forms under Fedosov product. The universal based linear map $A \rightarrow R A$ is the inclusion of $A$ as the forms of degree zero.

Suppose now that $A$ is a quasi-free algebra. We can then construct inductively a compatible family of lifting homomorphisms $A \rightarrow R A / I A^{n}$, and hence a lifting homomorphism

$$
l: A \rightarrow \widehat{R} A=\underset{n}{\lim } R A / I A^{n} .
$$

We can view $l$ as a universal lifting homomorphism in the following sense. Let $A=R / I$ be any nilpotent extension and choose a based linear lifting $\rho: A \rightarrow R$. Then $\rho$ extends uniquely to a homomorphism $\rho_{*}: \widehat{R} A \rightarrow R$, whose composition with $l$ gives a lifting homomorphism $A \rightarrow R$.

The first step in the construction of $l$ is to choose a lifting homomorphism $A \rightarrow R A / I A^{2}$. We have seen this is of the form $a \mapsto a-\phi a$, where

$$
\phi: \bar{A} \rightarrow \Omega^{2} A, \quad-\delta \phi=d \cup d .
$$

The succeeding steps seem at first glance to involve lots of choice. However, as we shall see, there is a systematic procedure motivated by Yang-Mills theory to construct $l$ starting from a choice of $\phi$. 
We begin by noting that a homomorphism $R A / I A^{n} \rightarrow R$ is equivalent to a based linear map $\rho: A \rightarrow R$, whose curvature $\omega$ satisfies $\omega^{n}=0$, where

$$
\omega^{n}\left(a_{1}, \ldots, a_{2 n}\right)=\omega\left(a_{1}, a_{2}\right) \cdots \omega\left(a_{2 n-1}, a_{2 n}\right) .
$$

A lifting homomorphism $A \rightarrow R A / I A^{n}$ is thus equivalent to a transformation $\rho \mapsto \rho^{\prime}$, natural in $R$, from such linear maps to homomorphisms $\rho^{\prime}: A \rightarrow R$, such that $\rho=\rho^{\prime}$ when $\rho$ is already a homomorphism. Therefore $l$ can be interpreted as a natural retraction which flattens based linear maps with nilpotent curvature into homomorphisms.

A flattening process of this sort is suggested by Yang-Mills theory. The gradient of the Yang-Mills functional is a vector field $X$ on the space of connections such that $-X$ decreases curvature. In good cases the associated flow $\exp (t X)$, in the limit as $t \rightarrow-\infty$, yields a retraction onto the subspace of flat connections.

We now look for an analogue of the Yang-Mills flow in our situation. A natural vector field on the space of based linear maps $\rho: A \rightarrow R$ for any $R$ is equivalent to a derivation $D: R A \rightarrow R A$. Because $R A$ is a free algebra, $D$ is determined by its restriction to $A$, which can be any linear map $A \rightarrow R A$ such that $D 1=0$. Let us write

$$
D a=f_{0} a+f_{1} a+\cdots
$$

with the 1-cochains $f_{n}: \bar{A} \rightarrow \Omega^{2 n} A$ to be determined. We take $f_{0}=0$, so that $D(R A) \subset I A$, and hence homomorphisms $A \rightarrow R$ are fixed under the flow. Next, the effect of $D$ on the universal curvature is

$$
\begin{aligned}
D\left(d a_{1} d a_{2}\right) & =D\left(a_{1} a_{2}-a_{1} \circ a_{2}\right) \\
& =D\left(a_{1} a_{2}\right)-a_{1} \circ D a_{2}-D a_{1} \circ a_{2} \\
& =D\left(a_{1} a_{2}\right)-a_{1} D a_{2}-D a_{1} a_{2}+d a_{1} d D a_{2}+d D a_{1} d a_{2} .
\end{aligned}
$$

To first order, i.e., modulo $I A^{2}$, this is $f_{1}\left(a_{1} a_{2}\right)-a_{1} f_{1}\left(a_{2}\right)-f_{1}\left(a_{1}\right) a_{2}$. If we take $f_{1}=\phi$, then we have

$$
D\left(d a_{1} d a_{2}\right) \equiv d a_{1} d a_{2} \quad\left(\bmod I A^{2}\right)
$$

which shows that $-D$ decreases the curvature when terms of second order and higher are ignored. Finally, we put $f_{n}=0$ for $n \geq 2$. There are many other possibilities which can be constructed from $\phi$, for example, we might take $f_{2}$ to be $\phi$ followed by $a_{0} d a_{1} d a_{2} \mapsto a_{0} \phi a_{2} \phi a_{1}$, but this choice is the simplest from the present viewpoint.

Thus our analogue of the Yang-Mills vector field is the derivation $D$ on $R A$ defined by

$$
D a=\phi a .
$$

We have $D(R A) \subset I A$ and

$$
D\left(d a_{1} d a_{2}\right)=d a_{1} d a_{2}+d a_{1} d \phi a_{2}+d \phi a_{1} d a_{2},
$$

so $D=1$ on $I A / I A^{2}$. As in the second proof of the tubular neighborhood theorem, $D=n$ on $I A^{n} / I A^{n+1}=\Omega^{2 n} A$, and the eigenspaces of $D$ on $\widehat{R} A$ 
provide an isomorphism of filtered algebras

$$
\widehat{\Omega}^{e v} A=\prod_{n} \Omega^{2 n} A \simeq \widehat{R} A .
$$

In particular the kernel of $D$ is a subalgebra of $\widehat{R} A$ which is mapped isomorphically onto $A$ by the canonical surjection $\widehat{R} A \rightarrow A$, so we obtain the desired universal lifting homomorphism $A \rightarrow \widehat{R} A$.

If $\rho: A \rightarrow R$ has nilpotent curvature, and $\rho_{*}: \widehat{R} A \rightarrow R$ is the induced homomorphism, then

$$
\rho_{t} a=\rho_{*} e^{t D} a
$$

gives the trajectory of $\rho$ under the Yang-Mills flow. It is clear that as $t \rightarrow-\infty$ the map $\rho_{t}$ becomes the homomorphism $A \rightarrow R$ given by composing $\rho_{*}$ with the lifting homomorphism from $A$.

We can give a formula for the isomorphism (48) as follows. Let $H$ denote the degree zero operator on even forms which is multiplication by $n$ on $\Omega^{2 n} A$, and let $L$ denote the operator of degree 2 given by

$$
L\left(a_{0} d a_{1} \cdots d a_{2 n}\right)=\phi a_{0} d a_{1} \cdots d a_{2 n}+\sum_{j=1}^{2 n} a_{0} d a_{1} \cdots d a_{j-1} d \phi a_{j} d a_{j+1} \cdots d a_{2 n} .
$$

Then $[H, L]=L$ and $D=H+L$, hence on $\widehat{\Omega}^{e v} A$ we have

$$
e^{-L} H e^{L}=H+e^{-L}\left[H, e^{L}\right]=H+\int_{0}^{1} e^{-t L}[H, L] e^{t L} d t=D .
$$

Consequently the isomorphism (48) is given by the operator $e^{-L}$, and the universal lifting homomorphism is

$$
l a=e^{-L} a=a-\phi a+\frac{1}{2} L \phi a-\cdots .
$$

We next show that the derivation $D$ on $R A$ extends to a derivation on $Q A$ commuting with the canonical involution $\gamma$. We identify $Q A$ with the superalgebra of all differential forms under Fedosov product. The two canonical embeddings of $A$ in $Q A$ are then $\theta a=a+d a, \theta^{\gamma} a=a-d a$.

Since $Q A=A * A$, a derivation $D$ on $Q A$ is specified by giving a derivation $D \theta: A \rightarrow Q A$ relative to $\theta$ together with a derivation $D \theta^{\gamma}: A \rightarrow Q A$ relative to $\theta^{\gamma}$. This means that

$$
D \theta\left(a_{1} a_{2}\right)=\theta a_{1} \circ D \theta a_{2}+D \theta a_{1} \circ \theta a_{2}
$$

and similarly for $D \theta^{\gamma}$. It is straightforward to check that

$$
D \theta a=\frac{1}{2} d a+\phi a+d \phi a, \quad D \theta^{\gamma} a=-\frac{1}{2} d a+\phi a-d \phi a
$$

are derivations relative to $\theta$ and $\theta^{\gamma}$ respectively. Thus we obtain a derivation on $Q A$ defined by

$$
\begin{aligned}
D a & =\phi a, \\
D(d a) & =\frac{1}{2} d a+d \phi a
\end{aligned}
$$

and it clearly commutes with $\gamma$. 
Writing $J$ for the canonical ideal $\mathbf{q} A$, we have $D=0$ on $Q A / J=A$, and $D=\frac{1}{2}$ on $J / J^{2}=\Omega^{1} A$. Thus $D=\frac{1}{2} n$ on $J^{n} / J^{n+1}=\Omega^{n} A$, and the eigenspaces of $D$ on $\widehat{Q} A$ provide an isomorphism of filtered algebras

$$
\widehat{\Omega} A=\prod_{n} \Omega^{n} A \simeq \widehat{Q} A={\underset{\varliminf}{\lim }}_{n} Q A / \mathbf{q} A^{n}
$$

extending (48).

Suppose now that $A$ is a separable algebra. In this case we know that any two liftings of $A$ in a nilpotent extension are conjugate. The universal version of this fact says that there is an invertible element $u \in \widehat{Q} A$ such that $u \theta u^{-1}=\theta^{\gamma}$. We are going to construct such an element $u$ in a fashion similar to the one used above to construct a lifting homomorphism $A \rightarrow \widehat{R} A$.

Because $A$ is separable, there is an element $Y \in \Omega^{1} A$ satisfying $d a=$ $[a, Y]$, and, as we have seen, there is even a canonical choice for $Y$. Let $D^{\prime}$ be the derivation on $Q A$ such that

$$
D^{\prime} \theta=[\theta, Y], \quad D^{\prime} \theta^{\gamma}=-\left[\theta^{\gamma}, Y\right],
$$

where the bracket is with respect to the product on $Q A$. As these are derivations relative to $\theta$ and $\theta^{\gamma}$ respectively, $D^{\prime}$ is well defined. Adding and subtracting, and using the fact that $Y$ is odd, we obtain

$$
\begin{aligned}
D^{\prime} a & =d a \circ Y-Y \circ d a=d a Y-Y d a, \\
D^{\prime}(d a) & =a \circ Y-Y \circ a=(a Y-d a d Y)-(Y a+d Y d a) \\
& =d a+d(d a Y-Y d a) .
\end{aligned}
$$

Now the 1-cochains $d a Y$ and $-Y d a$ both have coboundary $-d a_{1} d a_{2}$, hence so does $\phi a=\frac{1}{2}(d a Y-Y d a)$. Thus $D^{\prime}$ is the derivation on $Q A$ given by

$$
D^{\prime} a=2 \phi a, \quad D^{\prime}(d a)=d a+2 d \phi a .
$$

Thus for this choice of $\phi$ we have $D^{\prime}=2 D$, where $D$ is the derivation considered above. The eigenvalues of $D^{\prime}$ on $\widehat{Q} A$ are nonnegative integers.

We now construct the desired invertible element in $\widehat{Q} A$ by solving a differential equation. The following calculations take place inside $\widehat{Q} A$, so that products and inverses are meant with respect to Fedosov product; one can use the isomorphism (51) to work inside $\widehat{\Omega} A$ with the ordinary product if one wants.

Let $Y=\sum y_{n}, D^{\prime} y_{n}=n y_{n}$ be the eigenvector decomposition of $Y$ with respect to $D^{\prime}$; we have $y_{0}=0$ since $Y \in J$. Let $g=\sum g_{n}, D^{\prime} g_{n}=n g_{n}$ be the element of $\widehat{Q} A$ satisfying

$$
D^{\prime} g=-Y g
$$

and $g_{0}=1$. The components $g_{n}$ are determined by the recursion relation

$$
-n g_{n}=y_{1} g_{n-1}+\cdots+y_{n} g_{0},
$$


so $g$ is a well-defined invertible element of $\widehat{Q} A$. We have

$$
\begin{aligned}
D^{\prime}\left(g^{-1} \theta g\right) & =\left(-g^{-1} D^{\prime} g g^{-1}\right) \theta g+g^{-1} D^{\prime} \theta g+g^{-1} \theta D^{\prime} g \\
& =g^{-1}(Y \theta+[Y, \theta]-\theta Y) g=0
\end{aligned}
$$

and similarly $D^{\prime}\left(g \theta^{\gamma} g^{-1}\right)=0$. Hence $g^{-1} \theta g$ and $g \theta^{\gamma} g^{-1}$ are both homomorphisms from $A$ to the kernel of $D^{\prime}$. Since $g_{0}=1$, these two homomorphisms coincide with the lifting homomorphism $A \rightarrow \widehat{R} A$, and we obtain the desired conjugacy:

$$
g^{-2} \theta g^{2}=\theta^{\gamma} \text {. }
$$

Finally, we note that the derivation $D$, or $D^{\prime}$, on $Q A$ has a geometric interpretation similar to the one discussed in the case of $R A$. It corresponds to a natural vector field $X$ on the space of pairs of homomorphisms $\theta^{\prime}, \theta^{\prime \prime}$ from $A$ to a variable algebra $R$ such that $-X$ decreases the difference $\theta^{\prime}-\theta^{\prime \prime}$. The associated flow in the limit carries $\theta^{\prime}$ and $\theta^{\prime \prime}$ to the same homomorphism $\theta_{0}$. In the separable case the infinitesimal changes in $\theta^{\prime}$ and $\theta^{\prime \prime}$ are given by infinitesimal inner automorphisms by (52). Solving the differential equation (53) amounts to integrating these infinitesimal inner automorphisms to inner automorphisms conjugating $\theta^{\prime}$ and $\theta^{\prime \prime}$ to $\theta_{0}$.

\section{Connections}

Connections in right modules. Let $E$ be a right $A$-module, and consider the space $E \otimes_{A} \Omega A$ of $E$-valued forms. This is naturally a graded right module over $\Omega A$, and by means of this structure we can write the element $\xi \otimes \omega$ of $E \otimes_{A} \Omega A$ as simply $\xi \omega$.

Following Connes [Co] we define a connection on $E$ to be an operator $\nabla$ : $E \rightarrow E \otimes_{A} \Omega^{1} A$ satisfying the Leibniz rule

$$
\nabla(\xi a)=\nabla(\xi) a+\xi d a
$$

for $\xi \in E, a \in A$. The operator $\nabla$ then extends uniquely to an operator of degree one on $E \otimes_{A} \Omega A$ satisfying

$$
\nabla(\eta \omega)=(\nabla \eta) \omega+(-1)^{|\eta|} \eta d \omega
$$

for $\eta \in E \otimes_{A} \Omega A, \omega \in \Omega A$.

Consider for example a free right module $V \otimes A$, where $V$ is a vector space, and identify the forms having values in $V \otimes A$ by means of the canonical isomorphism

$$
V \otimes \Omega A=(V \otimes A) \otimes_{A} \Omega A .
$$

Then we have a canonical connection given by the operator $\nabla=1 \otimes d$ on $V \otimes \Omega A$.

As another example, suppose that the right module $E$ is a direct summand of $V \otimes A$, and let $i: E \rightarrow V \otimes A$ and $p: V \otimes A \rightarrow E$ be the inclusion and projection maps. Then on $E$ we have an induced connection, called the Grassmannian connection, which is given by the composition

$$
E \otimes_{A} \Omega A \stackrel{i}{\rightarrow} V \otimes \Omega A \stackrel{1 \otimes d}{\rightarrow} V \otimes \Omega A \stackrel{p}{\rightarrow} E \otimes_{A} \Omega A,
$$


where we also use $i, p$ to denote the natural extensions to module-valued forms. Thus with this notation the Grassmannian connection is

$$
\nabla=p(1 \otimes d) i
$$

We now consider $E$ as a quotient of a free right module in the standard way by means of the multiplication map $m: E \otimes A \rightarrow E, m(\xi \otimes a)=\xi a$. A right module map $s: E \rightarrow E \otimes A$ such that $m s=1$ then identifies $E$ with a direct summand of $E \otimes A$, so it determines a connection on $E$.

Proposition 8.1. By associating to $s$ the Grassmannian connection $\nabla=$ $m(1 \otimes d) s$ we obtain a one-one correspondence between right A-module maps $s: E \rightarrow E \otimes A$ which are sections of $m$ and connections $\nabla$ on $E$.

Since sections $s$ of this sort exist iff $E$ is projective, we obtain

Corollary 8.2. A right module has a connection iff it is projective.

The proposition implies in particular that any connection occurs as a Grassmannian connection. This result can be viewed as a noncommutative analogue of the theorem of Narasimhan-Ramanan on universal connections [NR].

To prove the proposition we consider the exact sequence of right $A$-modules

$$
0 \rightarrow E \otimes_{A} \Omega^{1} A \stackrel{j}{\rightarrow} E \otimes A \stackrel{m}{\rightarrow} E \rightarrow 0,
$$

where $j(\xi d a)=\xi a \otimes 1-\xi \otimes a$. Ignoring the right module structure, this exact sequence of vector spaces has a splitting given by the section $\xi \mapsto \xi \otimes 1$ of $m$. Now the splittings of a short exact sequence form an affine space which can be identified with the space of maps from the quotient space to the subspace once one has chosen a basepoint. In our case this means that there is a one-one correspondence between linear sections $s: E \rightarrow E \otimes A$ of $m$ and linear maps $\nabla: E \rightarrow E \otimes_{A} \Omega^{1} A$ given by

$$
s(\xi)=\xi \otimes 1-j(\nabla \xi) .
$$

But

$$
\begin{aligned}
s(\xi a)-s(\xi) a & =\xi a \otimes 1-\xi \otimes a-j\{\nabla(\xi a)\}+j\{\nabla(\xi) a\} \\
& =j\{-\nabla(\xi a)+\nabla(\xi) a+\xi d a\}
\end{aligned}
$$

and $j$ is injective, so $s$ is a right $A$-module map iff $\nabla$ is a connection.

Connections on bimodules. Let $E$ be a bimodule over $A$. By a right connection on $E$ we mean an operator $\nabla_{r}: E \rightarrow E \otimes_{A} \Omega^{1} A$ satisfying

$$
\nabla_{r}\left(a_{1} \xi a_{2}\right)=a_{1}\left(\nabla_{r} \xi\right) a_{2}+a_{1} \xi d a_{2} .
$$

In other words, a right connection is a connection on the right module $E$ which commutes with left multiplication.

Let $\nabla$ be a connection on $E$ as right module, and let $s$ be the corresponding section (55). Since

$$
s(a \xi)-a s(\xi)=j\{-\nabla(a \xi)+a \nabla \xi\},
$$

$\nabla$ commutes with left multiplication iff $s$ does. Thus a right connection $\nabla_{r}$ is equivalent to a bimodule map $s_{r}: E \rightarrow E \otimes A$ which is a section of the right multiplication map $m_{r}: E \otimes A \rightarrow E$. 
Similarly a left connection on $E$ is an operator $\nabla_{l}: E \rightarrow \Omega^{1} A \otimes_{A} E$ commuting with right multiplication and satisfying the Leibniz rule with respect to the left multiplication:

$$
\nabla_{l}\left(a_{1} \xi a_{2}\right)=a_{1}\left(\nabla_{l} \xi\right) a_{2}+d a_{1} \xi a_{2}
$$

It is equivalent to a bimodule map $s_{l}: E \rightarrow A \otimes E$ which is a section of the left multiplication map $m_{l}$.

Definition. A connection on the bimodule $E$ is a pair $\left(\nabla_{l}, \nabla_{r}\right)$ consisting of a left and a right connection.

To understand this concept better, let us consider the commutative square of bimodule surjections:

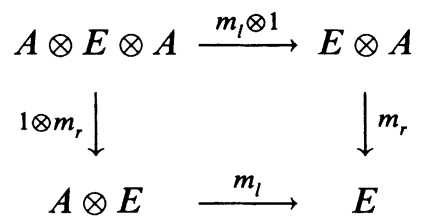

Given a connection $\left(\nabla_{l}, \nabla_{r}\right)$ on $E$, let $s_{l}, s_{r}$ be the corresponding sections as above. Then $\left(1 \otimes s_{r}\right) s_{l}$ is a lifting of the bimodule $E$ into $A \otimes E \otimes A$, showing that $E$ is a projective bimodule over $A$. Conversely, if $E$ is a projective bimodule, we can lift it into $A \otimes E \otimes A$ and then follow with $1 \otimes m_{r}, m_{l} \otimes 1$ to get the liftings $s_{l}, s_{r}$ corresponding to a connection on $E$. This proves

Proposition 8.3. A connection on $E$ exists iff $E$ is a projective bimodule over $A$.

Now a lifting $E$ into $A \otimes E \otimes A$ is equivalent to a connection on $E$ considered as a right module over $A^{e}$. In order to clarify the relation of these $A^{e}$-module connections to connections on the bimodule $E$ in our sense, we observe that the above square is part of the $3 \times 3$ diagram of short exact sequences obtained by tensoring the basic exact sequence (36) on both the left and right of $E$. This diagram yields in a well-known way an exact sequence of bimodules

$$
0 \rightarrow \Omega^{1} A \otimes_{A} E \otimes_{A} \Omega^{1} A \rightarrow A \otimes E \otimes A \rightarrow(A \otimes E) \times_{E}(E \otimes A) \rightarrow 0 .
$$

Since a connection on the bimodule $E$ is equivalent to a lifting of $E$ into the fibre product on the right, any connection of this type comes from an $A^{e}$ module connection on $E$. Moreover two $A^{e}$-module connections which give the same connection on $E$ differ by a bimodule map $E \rightarrow \Omega^{1} A \otimes_{A} E \otimes_{A} \Omega^{1} A$. We see therefore that $A^{e}$-module connection is a finer concept than connection in our sense.

Example. Suppose the algebra $A$ is separable, and let $Z=\sum x_{i} \otimes y_{i}$ be a separability element. We then have a bimodule direct sum decomposition

$$
\Omega^{1} A \underset{j}{\stackrel{p}{\leftrightarrows}} A \otimes A \underset{m}{\stackrel{s}{\leftrightarrows}} A,
$$

where $s(a)=a Z=Z a, p\left(a_{0} \otimes a_{1}\right)=a_{0} Y a_{1}$, and $Y=\sum x_{i} d y_{i}$.

On the free bimodule $A \otimes A$ we have the canonical connection given by $\nabla_{l}=d \otimes 1$ and $\nabla_{r}=1 \otimes d$. By virtue of this decomposition we obtain induced 
connections on $A$ and $\Omega^{1} A$. Now according to 4.1 there is in fact a canonical choice for the separability element $Z$. Consequently we find that for a separable algebra there are canonical connections on $A$ and $\Omega^{1} A$.

Let us now compute these connections for an arbitrary separability element $Z$. We first consider the case of $A$, where the induced connection is $\nabla_{l}=$ $m(d \otimes 1) s, \nabla_{r}=m(1 \otimes d) s$. We have

$$
\nabla_{l} a=m(d \otimes 1) \sum x_{i} \otimes y_{i} a=\sum d x_{i} y_{i} a=-Y a
$$

as $\sum x_{i} y_{i}=1$. A similar calculation holds for $\nabla_{r}$ yielding the formulas

$$
\begin{aligned}
& \nabla_{l} a=-Y a, \\
& \nabla_{r} a=Y a+d a=a Y .
\end{aligned}
$$

We next consider $\Omega^{1} A$, where the induced connection is $\nabla_{l}=p(d \otimes 1) j$, $\nabla_{r}=p(1 \otimes d) j$. Using $j\left(a_{0} d a_{1}\right)=a_{0} a_{1} \otimes 1-a_{0} \otimes a_{1}$, we find

$$
\begin{aligned}
& \nabla_{l}\left(a_{0} d a_{1}\right)=a_{0} d a_{1} Y+d a_{0} d a_{1}, \\
& \nabla_{r}\left(a_{0} d a_{1}\right)=-a_{0} Y d a_{1} .
\end{aligned}
$$

Extending derivations to $T_{A}(E)$. A connection on a vector bundle over a manifold provides a way to lift vector fields on the base to vector fields on the total space. We now derive a noncommutative version of this fact.

We consider an $A$-bimodule $E$ equipped with a connection $\left(\nabla_{l}, \nabla_{r}\right)$, and let $T=T_{A}(E)$. Given a derivation $D: A \rightarrow M$, where $M$ is a $T$-bimodule, we propose to extend $D$ to a derivation $T \rightarrow M$. It suffices to define a linear map $\xi \mapsto D \xi$ from $E$ to $M$ satisfying

$$
D\left(a_{1} \xi a_{2}\right)=D a_{1} \xi a_{2}+a_{1} D \xi a_{2}+a_{1} \xi D a_{2} .
$$

To see this recall that the derivation $D: T \rightarrow M$ we seek is equivalent to a lifting homomorphism $1+D: T \rightarrow T \oplus M$ into the semi-direct product. We are given this lifting homomorphism on $A$, hence by the universal property it suffices to arrange that $\xi \mapsto D \xi$ is a bimodule map relative to $a \mapsto a+D a$. This means

$$
a_{1} \xi a_{2}+D\left(a_{1} \xi a_{2}\right)=\left(a_{1}+D a_{1}\right)(\xi+D \xi)\left(a_{2}+D a_{2}\right)
$$

which is equivalent to $(60)$.

We define $D$ on $E$ to be the composition

$$
E \stackrel{\left(\nabla_{l}, \nabla_{r}\right)}{\longrightarrow} \Omega^{1} A \otimes_{A} E \oplus E \otimes_{A} \Omega^{1} A \stackrel{D_{*}}{\longrightarrow} M,
$$

where $D_{*}(d a \xi)=D a \xi$ and $D_{*}(\xi d a)=\xi D a$. Thus

$$
D \xi=D_{*} \nabla_{l} \xi+D_{*} \nabla_{r} \xi
$$

and (60) follows immediately from the properties (57), (56) of $\nabla_{l}, \nabla_{r}$. We have therefore proved the first part of 
Proposition 8.4. Any derivation $D: A \rightarrow M$, where $M$ is a bimodule over $T=T_{A}(E)$, extends to a derivation $D: T \rightarrow M$ given by (61). Consequently, the connection determines a bimodule splitting of the cotangent exact sequence

$$
0 \rightarrow T \otimes_{A} \Omega^{1} A \otimes_{A} T \rightarrow \Omega^{1} T \rightarrow T \otimes_{A} E \otimes_{A} T \rightarrow 0 .
$$

To obtain the second statement we consider the universal derivation from $A$ to a $T$-bimodule:

$$
A \stackrel{d}{\rightarrow} \Omega^{1} A \subset T \otimes_{A} \Omega^{1} A \otimes_{A} T
$$

and extend it to $T$; the bimodule map defined on $\Omega^{1} T$ associated to this extension is then a retraction which splits the sequence.

Connections on $\Omega^{1} A$. Let us next take the bimodule $E$ to be $\Omega^{1} A$. Since $\Omega^{1} A \otimes_{A} \Omega^{1} A=\Omega^{2} A$, a left connection $\nabla_{l}$ on $\Omega^{1} A$, a right connection $\nabla_{r}$, and $d$ can be viewed as operators from $\Omega^{1} A$ to $\Omega^{2} A$ having the properties

$$
\begin{aligned}
\nabla_{l}\left(a_{1} \xi a_{2}\right) & =a_{1} \nabla_{l} \xi a_{2}+d a_{1} \xi a_{2}, & \\
d\left(a_{1} \xi a_{2}\right) & =a_{2} d \xi a_{2}+d a_{1} \xi a_{2} & -a_{1} \xi d a_{2}, \\
\nabla_{r}\left(a_{1} \xi a_{2}\right) & =a_{1} \nabla_{r} \xi a_{2} & +a_{1} \xi d a_{2} .
\end{aligned}
$$

It is clear that if $\nabla_{r}$ is a right connection, then $d+\nabla_{r}$ is a left connection. Conversely if $\nabla_{l}$ is a left connection, then $\nabla_{l}-d$ is a right connection. Thus we have

Proposition 8.5. There is a one-one correspondence between left and right connections on $\Omega^{1} A$ given by $\nabla_{l}=d+\nabla_{r}$.

Consequently, if a right connection exists, then a left connection exists and conversely. In this case a connection exists, so $\Omega^{1} A$ is a projective bimodule by 8.3 , and $A$ is quasi-free. Conversely, if $A$ is quasi-free, then connections on $\Omega^{1} A$ exist. If $\left(\nabla_{l}, \nabla_{r}\right)$ is a connection, then

$$
\tau=\nabla_{l}-d-\nabla_{r}: \Omega^{1} A \rightarrow \Omega^{2} A
$$

is a bimodule map which will be called the torsion of the connection. It vanishes when $\nabla_{l}$ corresponds to $\nabla_{r}$ as in the proposition.

We next describe connections on $\Omega^{1} A$ by cochains.

Proposition 8.6. (1) A right connection $\nabla_{r}$ has the form

$$
\nabla_{r}\left(a_{0} d a_{1}\right)=a_{0} \phi a_{1},
$$

where $\phi a=\nabla_{r}(d a)$ can be any 1-cochain with values in $\Omega^{2} A$ satisfying $-\delta \phi=$ $d \cup d$.

(2) $A$ left connection $\nabla_{l}$ has the form

$$
\nabla_{l}\left(a_{0} d a_{1}\right)=a_{0} \psi a_{1}+d a_{0} d a_{1},
$$

where $\psi a=\nabla_{l}(d a)$ can be any 1-cochain with values in $\Omega^{2} A$ satisfying $-\delta \psi=$ $d \cup d$.

Proof. (1) follows from 3.4. (2) follows by applying (1) to the right connection $\nabla_{r}=\nabla_{l}-d$ corresponding to $\nabla_{l}$ in the sense of 8.5. 
A connection $\left(\nabla_{l}, \nabla_{r}\right)$ on $\Omega^{1} A$ is therefore described by the pair of $1-$ coohains $\psi a=\nabla_{l}(d a), \phi a=\nabla_{r}(d a)$ which can be arbitrary satisfying $-\delta \phi=$ $-\delta \psi=d \cup d$. The torsion is the bimodule map from $\Omega^{1} A$ to $\Omega^{2} A$ corresponding to the derivation $a \mapsto(\psi-\phi)(a)$. Thus the connection is torsion-free when $\phi=\psi$, and by 3.4 the torsion-free connections are in one-one correspondence with lifting homomorphisms $A \rightarrow R A / I A^{2}$.

One can always associate a torsion-free connection to a connection by averaging $\phi$ and $\psi$. This process has the virtue of preserving the geodesic flow, as we will see.

Examples. 1. Consider a free algebra $A=T(V)$. In this case $\Omega^{1} A=A \otimes$ $V \otimes A$ is the free bimodule generated by the vector space of differentials $d v$ for $v \in V$, and there is a canonical torsion-free connection with $\nabla_{l}(d v)=$ $\nabla_{r}(d v)=\phi v=0$. The corresponding lifting homomorphism $A \rightarrow R A / I A^{2}$ is the homomorphism extending the obvious lifting $v \mapsto v$ of the vector space of generators. A formula for $\phi$ is given at the end of $\S 3$.

2. Suppose $A$ separable and consider the connection (59) on $\Omega^{1} A$. We have $\phi a=\nabla_{r}(d a)=-Y d a$ and $\psi a=\nabla_{l}(d a)=d a Y$, and the torsion is the bimodule map corresponding to the derivation $a \mapsto d a Y+Y d a$. Thus the canonical connection on $\Omega^{1} A$ for a separable algebra is not torsion-free in general.

Geodesic flow and exponential map. We now discuss a noncommutative version of geodesic flow on the tangent bundle. Recall that a connection on the tangent bundle $T M$ of a manifold $M$ determines a vector field $X$ on $T M$ whose trajectories give the geodesics associated to the connection. At a point $v$ of $T M$ lying over the point $m \in M$ the vector $X_{v}$ is the horizontal tangent vector given by the connection which lifts $v$ regarded as a tangent vector to $M$ at the point $m$. Notice that the projection of $X$ to $M$ is tautological, more precisely, for a function $a$ coming from $M$ we have that $X a$ is the linear function on $T M$ corresponding to $d a$. Thus we have a canonical derivation from functions on $M$ to functions on $T M$, which is then extended to all functions on $T M$ by means of the connection.

As noncommutative analogue of the tangent bundle for an algebra $A$ we take the algebra $T_{A}\left(\Omega^{1} A\right)=\Omega A$. Thus $\Omega^{n} A$ corresponds to the space of functions of degree $n$ on the tangent bundle, and we have the canonical derivation $d$ : $A \rightarrow \Omega^{1} A$ with values in the space of linear functions. Suppose now that a connection $\left(\nabla_{1}, \nabla_{r}\right)$ on $\Omega^{1} A$ is given, and let $\psi, \phi$ be the associated cochains as above. We then use the connection as in 8.4 to extend $d$ to the geodesic flow derivation $X: \Omega A \rightarrow \Omega A$ defined by

$$
\begin{aligned}
X a & =d a, \\
X(d a) & =\left(\nabla_{l}+\nabla_{r}\right)(d a)=(\psi+\phi)(a) .
\end{aligned}
$$

We note that the geodesic flow derivation is the same if we replace our connection by the torsion-free connection obtained by averaging $\psi$ and $\phi$.

The derivation $X$ is of degree one relative to the grading on $\Omega A$, so $X$ can be exponentiated to a one-parameter group of automorphisms $e^{t X}$ on $\widehat{\Omega} A$. 
Let $u: Q A \rightarrow \widehat{\Omega} A$ be the homomorphism such that $\imath a \mapsto a, \imath^{\gamma} a \mapsto e^{X} a$. We consider the $\mathbf{q} A$-adic filtration on $Q A$ and the filtration by order on $\widehat{\Omega} A$. We have

$$
\begin{aligned}
& p a \mapsto \frac{1}{2}\left(1+e^{X}\right) a=a+\frac{1}{2} d a+2 \text { nd order, } \\
& q a \mapsto \frac{1}{2}\left(1-e^{X}\right) a=-\frac{1}{2} d a+2 \text { nd order, }
\end{aligned}
$$

so $u$ is a homomorphism of filtered algebras. Identifying the associated graded algebra of $Q A$ with $\Omega A$, the induced map of associated graded algebras is given by $a \mapsto a, d a \mapsto-\frac{1}{2} d a$. As this induced map is an isomorphism, we obtain an isomorphism of the $\mathbf{q} A$-adic completion $\widehat{Q} A$ with $\widehat{\Omega} A$. Thus we have proved

Proposition 8.7. There is an isomorphism $\widehat{Q} A \stackrel{\sim}{\rightarrow} \widehat{\Omega} A$ given by $\iota a \mapsto a, l^{\gamma} a \mapsto$ $e^{X} a$.

We call this isomorphism the formal exponential map associated to the connection, since it is analogous to the map $T M \rightarrow M \times M$ sending a tangent vector $v$ at $m \in M$ to the pair $\left(m, \exp _{m} v\right)$.

Connections on $\Omega^{n} A$. Our analysis of connections on $\Omega^{1} A$ generalizes in a straightforward way to connections on $\Omega^{n} A$. In this case $\nabla_{l}, \nabla_{r}, d$ are operators from $\Omega^{n} A$ to $\Omega^{n+1} A$, the torsion is the bimodule map $\nabla_{l}-d+(-1)^{n} \nabla_{r}$, and the one-one correspondence between left and right connections is given by $\nabla_{l}=d-(-1)^{n} \nabla_{r}$.

Proposition 8.8. (1) A right connection $\nabla_{r}$ on $\Omega^{n} A$ has the form

$$
\nabla_{r}\left(a_{0} d a_{1} \cdots d a_{n}\right)=a_{0} \phi\left(a_{1}, \ldots, a_{n}\right),
$$

where $\phi\left(a_{1}, \ldots, a_{n}\right)=\nabla_{r}\left(d a_{1} \cdots d a_{n}\right)$ can be any $n$-cochain with values in $\Omega^{n+1} A$ satisfying $(-1)^{n} \delta \phi=d^{\cup(n+1)}$.

(2) A left connection $\nabla$ on $\Omega^{n} A$ has the form

$$
\nabla_{l}\left(a_{0} d a_{1} \cdots d a_{n}\right)=a_{0} \psi\left(a_{1}, \ldots, a_{n}\right)+d a_{0} \cdots d a_{n},
$$

where $\psi\left(a_{1}, \ldots, a_{n}\right)=\nabla_{l}\left(d a_{1} \cdots d a_{n}\right)$ can be any $n$-cochain with values in $\Omega^{n+1} A$ satisfying $-\delta \psi=d^{\cup(n+1)}$.

Proof. The formula (63) gives an equivalence between linear maps $\nabla_{r}: \Omega^{n} A \rightarrow$ $\Omega^{n+1} A$ compatible with left multiplication and $n$-cochains $\phi$ with values in $\Omega^{n+1} A$. Using the identity (32) we have

$$
\nabla_{r}\left(a_{0} d a_{1} \cdots d a_{n} a_{n+1}\right)-\nabla_{r}\left(a_{0} d a_{1} \cdots d a_{n}\right) a_{n+1}=(-1)^{n} a_{0}(\delta \phi)\left(a_{1}, \ldots, a_{n+1}\right) \text {. }
$$

This shows $\nabla_{r}$ satisfies the Leibniz rule with respect to right multiplication iff $(-1)^{n} \delta \phi=d^{\cup(n+1)}$, proving (1).

(2) follows by applying (1) to the right connection $(-1)^{n+1}\left(\nabla_{l}-d\right)$.

A connection $\left(\nabla_{l}, \nabla_{r}\right)$ on $\Omega^{n} A$ is thus described by the pair of $n$-cochains $\psi=\nabla_{l} d^{\cup n}, \phi=\nabla_{r} d^{n^{r}}$ which can be arbitrary satisfying $-\delta \phi=(-1)^{n} \delta \psi=$ 
$d^{\cup(n+1)}$. The torsion is the bimodule map

$$
\nabla_{l}-d+(-1)^{n} \nabla_{r}: \Omega^{n} A \rightarrow \Omega^{n+1} A
$$

corresponding to the $n$-cocycle $\psi+(-1)^{n} \phi$.

For example, suppose $n=0$ in which case $A$ must be separable for connections to exist. A connection is described by elements $\psi=\nabla_{l} 1$ and $\phi=\nabla_{l} 1$ in $\Omega^{1} A$ satisfying $d a=-[a, \psi]=[a, \phi]$. The torsion $\psi+\phi$ is a central element of $\Omega^{1} A$. In the example (58), and in particular for the canonical connection on $A$, the torsion is zero.

\section{REFERENCES}

[B] D. Benson, Representations and cohomology. I, Cambridge Univ. Press, Cambridge, England, 1991.

[Co] A. Connes, Non-commutative differential geometry, Inst. Hautes Études Sci. Publ. Math. 62 (1985), 257-360.

[CC] A. Connes and J. Cuntz, Quasi homomorphismes, cohomologie cyclique et positivité, Comm. Math. Phys. 114 (1988), 515-526.

[Cu1] J. Cuntz, A new look at KK-theory, $K$-Theory 1 (1987), 31-51.

[Cu2] $\frac{}{5-8}$, Universal extensions and cyclic cohomology, C. R. Acad. Sci. Paris Sér. I 309 (1989),

[F] B. V. Fedosov, Analytical formulas for the index of an elliptic operator, Trudi Moskov. Mat. Obshch. 30 (1974), 159-241; English transl., Trans. Moscow Math. Soc. 30 (1974), 159-240.

[HKR] G. Hochschild, B. Kostant, and A. Rosenberg, Differential forms on regular affine algebras, Trans. Amer. Math. Soc. 102 (1962), 383-408.

[NR] M. S. Narasimhan and S. Ramanan, Existence of universal connections, Amer. J. Math. 83 (1961), 563-572.

[Ka] M. Karoubi, Homologie cyclique et $K$-théorie, Astérisque 149 (1987).

[Q] D. Quillen, Algebra cochains and cyclic cohomology, Inst. Hautes Études Sci. Publ. Math. 68 (1988), 139-174.

[Sh] W. F. Shelter, Smooth algebras, J. Algebra 103 (1986), 677-685.

Institute Of Mathematics, University Of Heidelberg, Im Neuenheimer Feld 288, 69120 HEIDELBERG, GeRMANY

E-mail address: cuntz@math.uni-heidelberg.de

University of Oxford, Mathematical Institute, 24-29 St. Giles, Oxford OX1 3LB, UNITED KINGDOM

E-mail address: quillen@maths.ox.ac.uk 